\title{
Stimulus-Specific Adaptation in the Gerbil Primary Auditory Thalamus Is the Result of a Fast Frequency-Specific Habituation and Is Regulated by the Corticofugal System
}

\author{
Peter Bäuerle, Wolfger von der Behrens, Manfred Kössl, and Bernhard H. Gaese \\ Institute for Cell Biology and Neuroscience, Department of Biological Sciences, Goethe University, D-60323 Frankfurt am Main, Germany
}

The detection of novel and therefore potentially behavioral relevant stimuli is of fundamental importance for animals. In the auditory system, stimulus-specific adaptation (SSA) resulting in stronger responses to rare compared with frequent stimuli was proposed as such a novelty detection mechanism. SSA is a now well established phenomenon found at different levels along the mammalian auditory pathway. It depends on various stimulus features, such as deviant probability, and may be an essential mechanism underlying perception of changes in sound statistics. We recorded neuronal responses from the ventral part of the medial geniculate body (vMGB) in Mongolian gerbils to determine details of the adaptation process that might indicate underlying neuronal mechanisms. Neurons in the vMGB exhibited a median spike rate change of $15.4 \%$ attributable to a fast habituation to the frequently presented standard stimulus. Accordingly, the main habituation effect could also be induced by the repetition of a few uniform tonal stimuli. The degree of habituation was frequency-specific, and comparison across simultaneously recorded units indicated that adaptation effects were apparently topographically organized. At the population level, stronger habituation effects were on average associated with the border regions of the frequency response areas. Finally, the pharmacological inactivation of the auditory cortex demonstrated that SSA in the vMGB is mainly regulated by the corticofugal system. Hence, these results indicate a more general function of SSA in the processing and analysis of auditory information than the term novelty detection suggests.

\section{Introduction}

It is a common feature of neuronal information processing that repetitive stimulation results in reduced neuronal activation. This can be functionally significant in the form of stimulusspecific adaptation (SSA), a decrease in neuronal responses only to the repeated stimulus, which is not generalized to other stimuli (Nelken and Ulanovsky, 2007). Neurons showing strong SSA were recently described at different levels of the central auditory pathway (Ulanovsky et al., 2003; Pérez-González et al., 2005; Antunes et al., 2010). They are seen as "novelty detectors" showing preferential responses to rare, unexpected acoustic stimuli among frequently occurring, steady background signals. The main factor influencing neuronal activity of those cells with the potential to detect "novel events" is the parameter of deviance between the two types of stimuli. In most studies, this parameter was the frequency separation between pure tones (Nelken and Ulanovsky, 2007). However, sound amplitude and binaural cues have successfully been tested as well (Reches and Gutfreund, 2008).

\footnotetext{
Received Nov. 4, 2010; revised April 27, 2011; accepted May 1, 2011.

Author contributions: P.B. and B.H.G. designed research; P.B. performed research; P.B. analyzed data; P.B., W.v.d.B., M.K., and B.H.G. wrote the paper.

This work was supported by Deutsche Forschungsgemeinschaft (P.B., W.v.d.B.) and Vereinigung von Freunden und Förderern der Johann Wolfgang Goethe-Universität (P.B.).

Correspondence should be addressed to Peter Bäuerle, Institute for Cell Biology and Neuroscience, Department of Biological Sciences, Goethe University, Siesmayerstrasse 70A, D-60323 Frankfurt am Main, Germany. E-mail: peter.baeuerle@gmx.de.

DOI:10.1523/JNEUROSCI.5814-10.2011

Copyright $\odot 2011$ the authors $\quad 0270-6474 / 11 / 319708-15 \$ 15.00 / 0$
}

Although the notion of novelty detection focuses on a very general, widely applicable concept, neurons with strong SSA are in fact only a fraction of the neurons exhibiting SSA at all. A substantial number (Ulanovsky et al., 2003; Malmierca et al., 2009; Antunes et al., 2010) or even the predominant fraction (Anderson et al., 2009; von der Behrens et al., 2009) of neurons tested showed rate adaptation only to a limited degree, suggesting that neuronal processes underlying SSA might be important for additional aspects of sensory information processing. The auditory thalamus [medial geniculate body (MGB)] is of particular interest in this respect, because SSA is not distributed uniformly across its subdivisions. Although Ulanovsky et al. (2003) found no effects at the level of the cat MGB, SSA was recently demonstrated throughout the mouse (Anderson et al., 2009) and rat MGB (Antunes et al., 2010). Both studies found relatively strong SSA in the medial division, whereas neurons in the ventral division (vMGB) showed adaptation to a minor degree. Whereas Anderson et al. (2009) found no SSA in a smaller sample of dorsal division neurons, Antunes et al. (2010) reported also strong SSA for this subdivision.

Attempts to understand adaptive information processing in the vMGB must take into account two main characteristics of this area: first, it is tonotopically organized, and second, it operates under powerful cortical control, via both direct and indirect corticofugal projections. It is well known that the auditory cortex adjusts and improves signal processing at subcortical stages through frequency-dependent facilitation and inhibition acting on different timescales from short-term changes to long-term 
plasticity (He, 2003a; Yan et al., 2005; Winer, 2006; Suga, 2008). This makes it rather likely that the strong effects of SSA in the auditory cortex are relayed to the vMGB directly or via corticofugal projections to the inferior colliculus (IC) and the thalamic reticularis nucleus (TRN) in which, again, SSA has already been described (Malmierca et al., 2009; Yu et al., 2009).

Therefore, we investigated three main aspects of SSA at the level of the vMGB: the time course of adaptation, frequencyspecific effects, and possibly underlying mechanisms. The hypothesis that SSA is relayed onto the vMGB via the corticofugal system was finally tested at the network level by inactivating auditory cortical structures.

\section{Materials and Methods}

Surgical procedure. Experiments were performed on 27 adult Mongolian gerbils (Meriones unguiculatus, 15 males, 12 females; weighting 44-75 g; mean of $58 \mathrm{~g}$ ) with clean outer ear canals. Animals were anesthetized by a mixture of $50 \mathrm{mg} / \mathrm{ml}$ ketamine hydrochloride (Ketavet; Pfizer) and 2\% xylazine hydrochloride (Rompun; Bayer). We induced anesthesia by an initial intramuscular injection of $2.1 \mathrm{ml} / \mathrm{kg}$ body weight $(\sim 0.12 \mathrm{ml})$, followed by subsequent injections of $0.9 \mathrm{ml} / \mathrm{kg}(\sim 0.05 \mathrm{ml})$ as needed. As soon as the animal was deeply anesthetized, a continuous subcutaneous injection administered by a micro pump (Genie; Kent Scientific) maintained a surgical level of anesthesia (dose rate at a level $\sim 46 \mu \mathrm{l} / \mathrm{h}$ ). After completing the surgical procedure and before starting with the electrode positioning, we lowered the dose rate to a level $\sim 36 \mu \mathrm{l} / \mathrm{h}$. Breathing rate, pedal-withdrawal reflex, and spontaneous neuronal activity were monitored to ensure a sufficient and constant level of anesthesia. A subcutaneous injection of $2 \mathrm{ml}$ of isotonic solution (Normofundin; Braun) created a liquid depot to stabilize the animal throughout the experiment. Dexamethasone sodium phosphate $(20 \mathrm{mg} / \mathrm{kg}$, i.p.; Sigma) was applied to prevent brain edema (Stiebler et al., 1997), and the eyes were covered with eye and nasal ointment (Bepanthen; Roche) to prevent drying out. The body temperature was maintained by a heating pad $\left(37.8^{\circ} \mathrm{C}\right)$.

Before surgery, the local anesthetic lidocaine (Xylocaine 2\%; Astra Zeneca) was applied subcutaneously to the upper part of the skull of the anesthetized animals. The skin was opened, and a head holder was attached to the skull using super glue and dental cement (Haraeus Kulzer). The skull and the electrode holder were aligned to allow for electrode movements in parasagittal planes, and bregma and lambda were leveled in the horizontal plane. Furthermore, the roll rotation of the head was adjusted by choosing two reference points on the skull $1 \mathrm{~mm}$ from the sagittal midline to each side (on a plain part of the skull). A vertical height disparity of no more than $30 \mu \mathrm{m}$ was accepted (Mitutoyo digimatic 164-171, accuracy of $2 \mu \mathrm{m}$ ). We performed a rectangular craniotomy (typically $2.9-5.2 \mathrm{~mm}$ posterior and $2.4-3.9 \mathrm{~mm}$ lateral to bregma) above the left brain hemisphere to provide vertical access to the MGB and removed the dura mater. In addition, a centrally located hole was drilled in the parietal bone above the right cerebral hemisphere and a silver/ silver-chloride reference electrode was placed between dura mater and the skull. In experiments with cortical inactivation, the left auditory cortex was exposed in a second craniotomy. The dura mater was removed above the cortex, and all exposed brain tissue including the hole for the reference electrode was covered with $20 \%$ gelatin to prevent drying out. Experimental procedures were approved by the local animal care committee and in full compliance with federal regulations as well as the Guide for the Care and Use of Laboratory Animals (National Research Council, 1996).

Electrophysiological recordings. Extracellular recordings in the MGB were made with a 16 -channel silicon multielectrode (16 recording sites spaced vertically $50 \mu \mathrm{m}$ apart with an approximate diameter of $15 \mu \mathrm{m}$ each; impedance, 1-3 M $\Omega$; model Alx16-5mm50-177; NeuroNexus Technologies). Advancement of the probe was interrupted by regular stops to allow the tissue to settle down. We started to record neuronal activity before we reached the target area. Once we recorded stimulusevoked spiking activity at the tip channel (probed with white noise, $50 \mathrm{~dB}$ SPL, rate of $1 \mathrm{~Hz}, 50 \mathrm{~ms}$ duration), the electrode was advanced with a step size of 10-20 $\mu \mathrm{m}$ (piezo electronic micromanipulator; Märzhäuser). To record from the ventrally located primary auditory thalamus, the electrode was lowered until stimulus-evoked spiking activity at the tip channel started to vanish again. Altogether, it took $>1 \mathrm{~h}$ to finally position the multielectrode within the MGB. For the experiments with cortical inactivation, local field potentials (LFPs) were recorded in the auditory cortex with a tungsten electrode (WE20023.0A3; MicroProbe) oriented approximately perpendicular to the cortical surface and positioned at a depth of $1000 \mu \mathrm{m}$ in layers V/VI. The neuronal signals were preamplified, filtered, and digitalized with a sampling rate of $24.4 \mathrm{kHz}$ [Tucker-Davis Technologies (TDT) System 3 modules: high-impedance head stage model RA16AC; Medusa preamplifier model RA16PA; two Medusa base stations model RA16BA]. The whole experiment, i.e., data acquisition and stimulus control, was done using the Brainware software package (TDT). Signals from the electrode were bandpass filtered (spikes, $600-3000 \mathrm{~Hz}$; local field potentials, $2-100 \mathrm{~Hz}$ ), and the raw voltage traces were stored for later offline analysis with Matlab (data import: damFileRead.m, Jan Schnupp, Oxford University, Oxford, UK). Recording positions were confirmed based on electrolytic lesions in nine experiments. After lesioning, animals were transcardially perfused $(0.9 \%$ saline, followed by $4 \%$ paraformaldehyde in buffer), the brain was removed, postfixed overnight, saturated in 10,20, and 30\% sucrose, sectioned on a freezing microtome (50 $\mu \mathrm{m}$ sections), and stained with cresyl violet (Nissl).

Auditory stimuli. All experiments were performed in a sound-attenuating chamber. The Brainware software (TDT) controlled the generation of auditory stimuli (5 ms cosine-squared rise/fall times) in real-time using TDT hardware (Realtime Processor RP21, System 3). Stimuli were attenuated (PA5; TDT System 3 ) to the desired SPL, then fed to a hi-fi amplifier (Grun$\operatorname{dig}$ SR1000), and presented to the right ear under free-field conditions (Sony loudspeaker SS-MS835). The sound system was calibrated with a $1 / 4$-inch Brüel \& Kjær microphone (model 4939).

Frequency response areas (FRAs) were measured at the beginning of each experiment using pure tones ( $50 \mathrm{~ms}$ duration) ranging from $600 \mathrm{~Hz}$ to $38,400 \mathrm{~Hz}$ with logarithmically spaced frequency steps of 0.25 octaves and sound intensities between -10 and $70 \mathrm{~dB}$ SPL in $10 \mathrm{~dB}$ steps. All frequency/intensity combinations were presented in random order (repetition rate of $1 \mathrm{~Hz}, 10$ averages). A tuning curve based on the raw spike data was immediately calculated afterward (some examples are given in Fig. $2 B$ ) to find the optimal frequencies for the adaptation paradigm located inside the maximal number of recorded frequency response areas across all channels within the vMGB. To test the stability of electrophysiological recording, we also determined the frequency response curve (FRC) at $60 \mathrm{~dB}$ SPL before and after the test protocol of a stimulus paradigm using the same frequencies as for the frequency response area $(600-38,400 \mathrm{~Hz})$.

Oddball paradigm. Our oddball design for determining frequencydependent adaptation consisted of repetitive stimulation (repetition rate, $2 \mathrm{~Hz})$ with tones of two frequencies separated by one octave $\left(f_{1}\right.$ and $f_{2}$; tone duration, $100 \mathrm{~ms}$ ) at a sound intensity of $60 \mathrm{~dB}$ SPL. In a "frozen" trial sequence of 100 stimuli, one tone was first used as the frequent standard stimulus ( $p=90 \%$ ) and the other one as the low-probability deviant $(p=10 \%)$ that was interspersed in a pseudorandomized order (Fig. $1 A$, bottom). The frozen trial sequence of 100 stimuli with deviants being presented at fixed positions (Fig. $1 \mathrm{~A}$, ts 1 ) was repeated 10 times to form a test block of 1000 stimuli (Fig. 1 A, B1). After a 2 min break at the end of this test block, another test block was presented in which the frequencies of deviant and standard were swapped (Fig. 1A, ts2, B2). This procedure was repeated five times to sample 500 single trials per given frequency in the deviant and in the preceding standard position. A complete stimulus set for testing two frequencies with an oddball design consisted of those five repetitions (10 test blocks) together with two intermediate control blocks with equal probability (50\%) for both frequencies and, as an additional control, a block of 100 pure tones (repetition rate $1 \mathrm{~Hz}$ ) for each stimulus frequency at the beginning and at the end of the stimulus set.

Reduced oddball paradigm. To resolve frequency-specific differences in adaptation on a finer scale, we used a "reduced oddball" design that included several frequency pairs tested with fewer repetitions. Standard and deviant stimuli in each frequency pair were presented in the same 
A

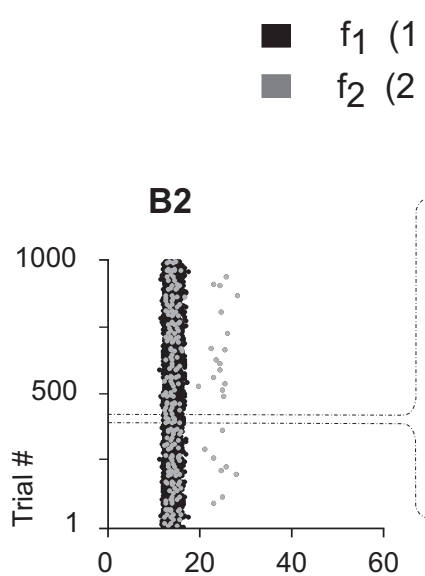

B1

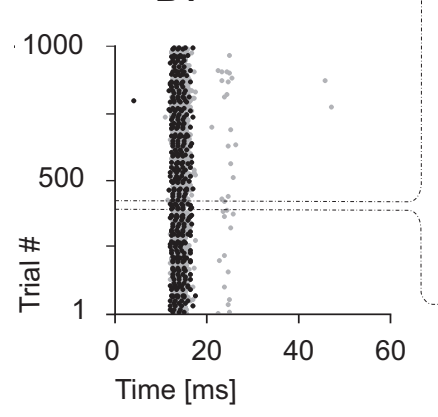

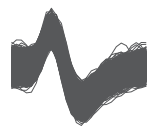

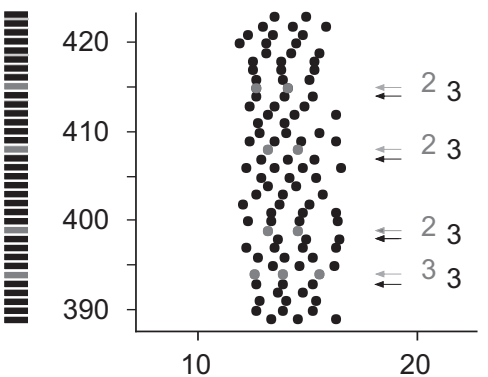

20
B

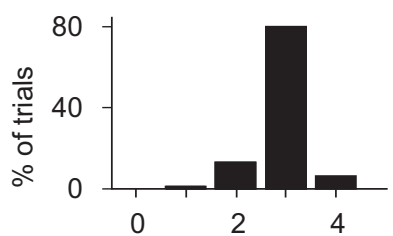

$f_{1}$ standard

$f_{2}$ deviant
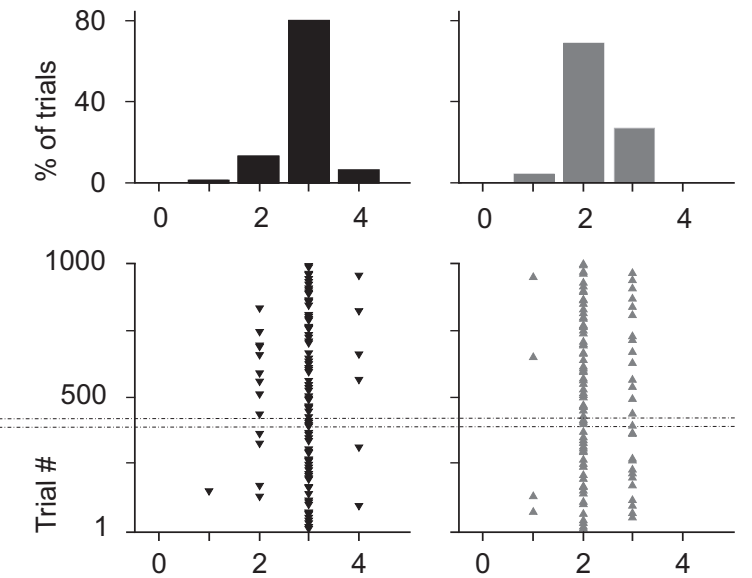

$f_{1}$ deviant

$f_{2}$ standard
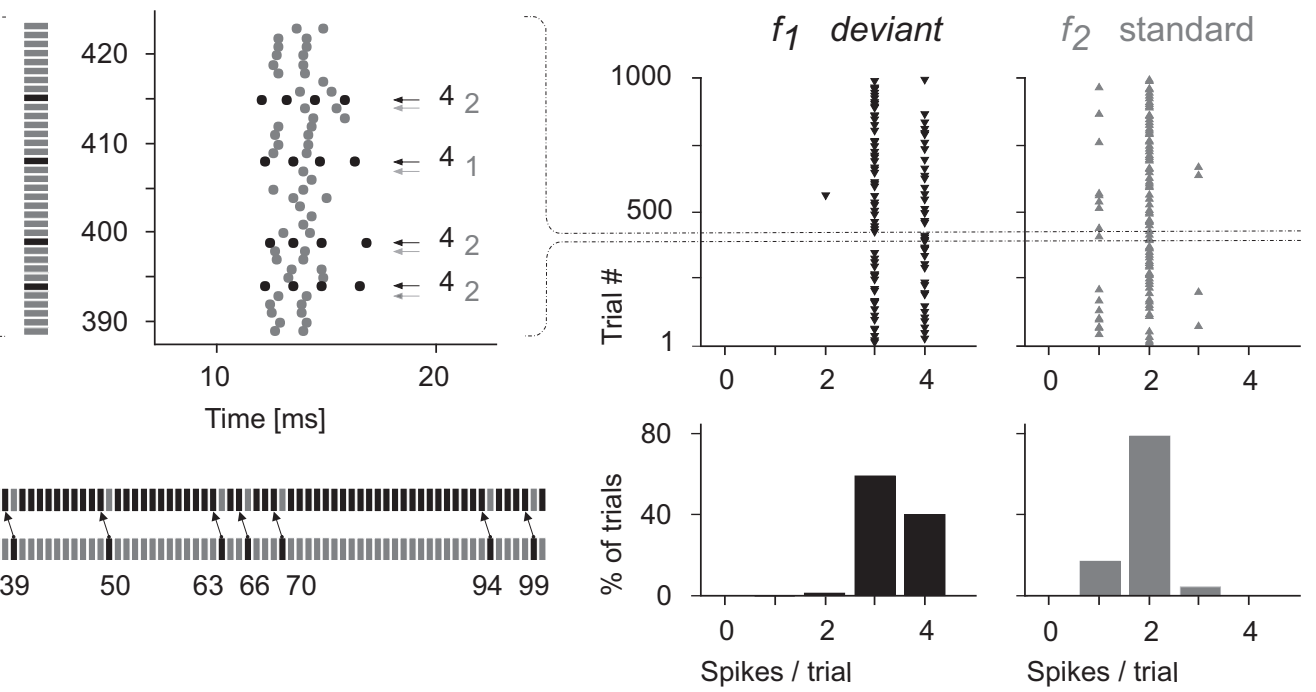

9499

Spikes / trial

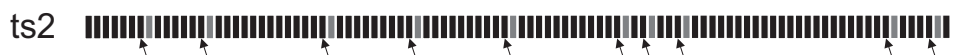

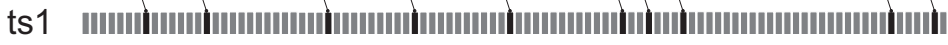

$\begin{array}{llllllll}8 & 15 & 29 & 39 & 50 & 63 & 66 & 70\end{array}$

Figure 1. Frequency-dependent adaptation in the spike rate of a well isolated vMGB unit. $\boldsymbol{A}$, The frozen trial sequence of 100 stimuli with fixed (pseudorandomized) deviant positions is illustrated in the bottom two lines (ts1, ts2). A frozen trial sequence consisted of $10 \%$ deviant and $90 \%$ standard stimuli with the two tones $f_{1}$ (here $1 \mathrm{kHz}$, black bars) and $f_{2}$ (here $2 \mathrm{kHz}$, gray bars) being the deviant in one and the standard in the other trial sequence. As indicated by small arrows, deviant-related responses of one frozen trial sequence (e.g., from ts1) were compared with each standard-related response in the preceding position of the corresponding sequence. A frozen trial sequence (e.g., ts 1 or ts2) was repeated 10 times comprising a block of 1000 trials. The responses of a single unit to two corresponding blocks B1 and B2 are shown in the middle and top parts of $A$. The dot plots indicate the timing of single spikes in the $60 \mathrm{~ms}$ after stimulus onset for all 1000 trials (left column) and a subset of 35 trials at a higher temporal resolution (right column). Responses to tones of frequency $f_{1}$ are indicated in black dots and responses to frequency $f_{2}$ in gray dots. Higher spike counts per trial can be observed for the frequency $f_{1}$ (black arrows, black numbers to the right) compared with the frequency $f_{2}$ (gray arrows, gray numbers to the right), regardless of $f_{1}$ being either the deviant in block B1 or the standard in block B2. However, focusing on the single frequency $f_{1}$, higher spike counts per trial can be noticed for $f_{1}$ as deviant (bottom row) than for $f_{1}$ as standard tone (top row). The same is true for $f_{2}$ being the deviant in the top row and the standard frequency in the bottom row. Spike waveforms of a $10 \%$ sample of all recorded responses to standard and deviant stimuli of both frequencies within this experiment are shown at the top right ( 4671 single waveforms). $\boldsymbol{B}$, Distribution of spike counts in responses to both frequencies $f_{1}$ and $f_{2}$ being either the standard (bottom, $f_{2}$; top, $f_{1}$ ) or the deviant frequency (bottom, $f_{1}$; top, $f_{2}$ ). Spike counts per trial are shown at a single-trial resolution ( 4 plots in the middle) or as the average distribution of spike counts (top and bottom). There is a noticeable shift from higher to lower spike counts in responses to both frequencies in the deviant with respect to the corresponding standard position.

pseudorandomized order of the frozen trial sequence as in the complete oddball paradigm. Each pair consisted of two pure tones separated by one octave, and one block consisted of two frequency pairs separated by 0.5 octaves (repetition rate, $2 \mathrm{~Hz}$ ). The first frequency pair was presented in the first 200 trials, the second frequency pair in the next 200 trials. Then the 400 trial sequence was repeated with swapped deviant and standard frequencies. This procedure was repeated for a total of four different frequency pairs as shown, for example, in Figure 7, including a 2 min break between blocks. Because we wanted to collect 200 deviant-tocorresponding-standard comparisons per given frequency, the whole stimulus arrangement was repeated 10 times to form a stimulus set in this protocol.

Roving oddball paradigm. In the roving oddball design, stimulus trains of five identical pure tones (one given frequency; duration, 100 $\mathrm{ms}$; sound intensity, $60 \mathrm{~dB}$ SPL; repetition rate, $2 \mathrm{~Hz}$ ) were presented.
A frozen trial sequence consists of 11 stimulus trains with frequencies spaced by 0.25 octaves covering a total frequency range of 2.5 octaves that matched the frequency tuning across all recording channels most suitable. Frequencies were numbered in ascending order and presented in the fixed order of frequency numbers starting with the following: $7,7,7,7,7,11,11,11,11,11,5,5,5, \ldots$ The fixed presentation order $(7,11,5,1,6,10,4,9,3,8,2)$ ensured a frequency step of at least one octave between consecutive trains. In the context of SSA, the first stimulus of each train was considered a "deviant stimulus" (new), whereas the last one (fifth) was taken as "standard stimulus" (Garrido et al., 2009). The frozen sequence was repeated 12 times to form a stimulus block of 660 stimuli, followed by a $2 \mathrm{~min}$ break. We used a stimulus set of 20 blocks resulting in a total of 240 single stimuli per given frequency in the first and the fifth position for the roving oddball protocol. 
Data analysis. Data analysis was performed in Matlab (version 7.6; MathWorks). Spike sorting was performed on the basis of the spike waveforms. All waveforms of one experiment were sorted together for each channel. The first five principal components of the waveforms were computed (Matlab function princomp) and clustered by an unsupervised classification algorithm [KlustaKwik (Kenneth D. Harris, Rutgers University, Newark, NJ); restricted to five clusters]. We generally recorded from small clusters of neurons (multiunit activity) rather than singleneuron activity and gained only in rare cases a well isolated spike cluster (one example is given in Fig. 1). Because comparable effects of SSA were reported for single-unit and multiunit activity recorded from a given brain area (Ulanovsky et al., 2003; Anderson et al., 2009; von der Behrens et al., 2009), we expected no bias for our data. Adaptation effects were quantified in the oddball paradigm, by determining the percentage difference between deviant- and standard-related response activity (numbers of spikes) as ((deviant - standard)/standard $\left.{ }^{\star} 100\right)$ named "DS difference" (in percent), which gives a concrete impression of the effects investigated here. To provide comparability to the literature, we also calculated the normalized spike adaptation index (SI) (stated in brackets within the text) (Ulanovsky et al., 2003). SI is calculated as (deviant standard)/(deviant + standard). The SI index ranges from -1 for a complete absence of deviant-related activity to +1 for a complete absence of standard-related activity.

Like Ulanovsky et al. (2004), we used a combined threshold criterion to define the frequency response area. We calculated the mean plus $1 \mathrm{SD}$ of the interstimulus activity level directly preceding stimulus onset (100 ms time window) and added $10 \%$ of the maximum spike activity of all frequency/level combinations to determine threshold activity. The neuronal frequency response area (tuning curve) was defined by linear interpolation of threshold activity in the frequency/level response matrix (Matlab function contour) (Abel and Kössl, 2009). Only the responses to stimulation frequencies within this tuning curve were further analyzed, and for the oddball paradigm, both frequencies of a stimulation pair had to be enclosed.

Our strategy was to focus on average changes in activity in large samples (many repetitions) to level out all spontaneous disparities and therefore reveal even small effects with statistical significance. As a drawback, this resulted in a rather long recording time (e.g., a complete test of the oddball protocol plus all controls and the two frequency response curves took almost $3 \mathrm{~h}$ ). Consequently, great care was taken to ensure stable recording conditions. Neuronal data were selected by means of the following criteria. (1) Spike sum activity calculated separately for deviant and standard tones of each frequency should not alter in an unsystematic manner across the blocks of the oddball sequences. For example, a change in overall spike sum activity for the deviants as a consequence of a slowly changing level of anesthesia should also be reflected by the sum of the corresponding standard tones. (2) We plotted the frequency response curves, calculated the area under the curve before and after each stimulus set, and excluded all units with a total area change of $>30 \%$. Furthermore, (3) we included only units with a clear V-shaped tuning curve, and (4) we analyzed only responses with a well defined onset component when tested with a block of 100 pure tones for the oddball paradigm. Because this last criterion is a qualitative description in the first place, we requantified it based on the oddball results: average stimulus-evoked activity in the first $30 \mathrm{~ms}$ time window had to be at least one spike/three trials (standard tone) for a unit to be included in our samples for the reduced oddball and the roving oddball design. We analyzed responses from an average of eight channels per experiment fulfilling all the criteria stated above.

Because of technical limitation of our multichannel recording at a repetition rate of $2 \mathrm{~Hz}$, we were restricted to acquire data only for $60 \mathrm{~ms}$ after stimulus onset. According to Anderson et al. (2009), this is sufficient to gather the typical transient neuronal response throughout the MGB. We further limited this time window to the first $30 \mathrm{~ms}$ of stimulusevoked neuronal response, which encompasses the phasic onset response in vMGB neurons (Fig. 2C). The latency was determined from the summed peristimulus time histogram (PSTH) with a bin size of $1 \mathrm{~ms}$.

The oddball paradigm included two different pure tone stimuli of two frequencies with very different stimulus probabilities (deviant, $p=0.1$ /
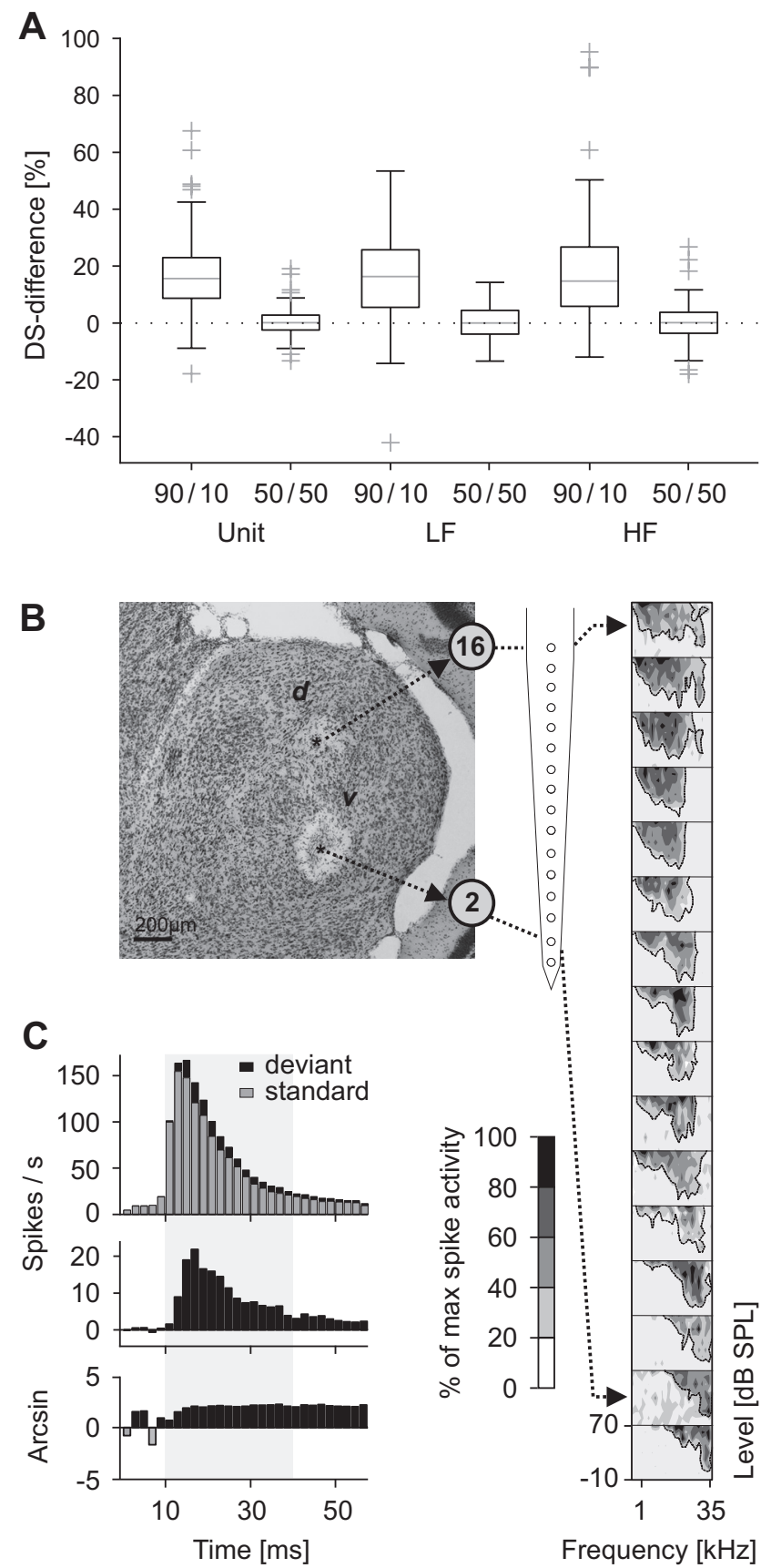

Frequency $[\mathrm{kHz}]$

Figure 2. Population analysis of unit activity recorded from the gerbil vMGB in the oddball paradigm ( $n=100) . A$, Differences in spike sum between responses to deviant and standard tones (DS difference) are shown. Beside the dataforthewhole population with units averaged across configurations B1 and B2 ("Unit," left2 boxplots), data werealso calculated separately for the relative LF(middle 2 box plots) and the relative HF (right 2 box plots) of each stimulation pair. For each separate population, data are given for the deviant probability of thetestcondition (90/10) as well asthe control condition (50/50).B, Coronal histologicalsection through the gerbil MGBstained with cresyl violet. Scalebar, $200 \mu \mathrm{m}$. v, Ventral part of the MGB; d, dorsal part of the MGB. Asterisks mark electrolytic lesions through recording channels 2 and 16 . A schematic drawing of the recording electrode together with the simultaneously recorded receptive field areas of all 16 channels calculated with the raw spike data (experimental control) is shown on the right side. The normalized level of activity per channel coded in grayscale is displayed to the left. This multichannel recording illustrates the tonotopic organization of the vMGB as well as the border to the dorsal division. In this example, the border was determined between channels 13 and 14 , indicated by a loosening of the tonotopic axes together with a widening of the neuronal receptive field areas on the dorsal side. $C$, Average onset response of vMGB units from the deviant compared with the standard configuration shown as population PSTH (top; averageactivity of 200 frequencies, bin size of $2 \mathrm{~ms}$ ), as averaged difference in spike activity (middle; deviant - standard), and as arc-sinus-corrected difference (bottom). Deviant-related activity is shown in black and standardrelated activity in gray. The time range with a light gray background illustrates the typical investigated $30 \mathrm{~ms}$ time window exemplified here for the population PSTH. 
standard, $p=0.9$ ). To compensate for this strong imbalance in trial numbers, we compared the evoked spike activity from the deviant positions with the activity from the directly preceding standard positions of the swapped blocks. The time course of neuronal responses was analyzed by arranging the evoked spike sum activities of all standard and deviant stimuli in the order of presentation. In this analysis, spike rates were normalized to the maximum evoked spike activity per trial for a given frequency and unit. Thus, normalized values taken from the time course analysis could not be directly compared with the DS differences.

Statistical analysis was performed using the Matlab statistics toolbox (version 5.1; MathWorks). Because parts of our data were not normally distributed, we applied only nonparametric tests to determine statistical differences. Statistical tests were considered significant at $p<0.05$. The term "highly significant" was used at $p<0.001$. Average population data are shown as median with the interquartile range displayed from 25 th to 75th percentile.

Cortical inactivation. The $\mathrm{GABA}_{\mathrm{A}}$ agonist muscimol decreases spike activity in a concentration-dependent manner with full efficiency (Rijal and Gross, 2008). The maximal spread of muscimol takes place within the first 30 min (Martin, 1991; Martin and Ghez, 1999; Talwar et al., 2001). It remains in the tissue (Martin and Ghez, 1999), and, applied cortically, it does not reach subcortical structures (Allen et al., 2008). It has been shown by Talwar et al. (2001) that, in the rat, a topical muscimol application of $20 \mu \mathrm{g}$ inhibited the auditory cortex, including the infragranular layers. However, as demonstrated by Happel et al. (2010) for the Mongolian gerbil, lower doses are also effective.

We used a total of $16 \mu \mathrm{g}$ of muscimol ( $1 \mu \mathrm{g}$ of muscimol dissolved in $1 \mu \mathrm{l}$ of physiological saline; Sigma-Aldrich) for inactivation of the auditory cortex. Before muscimol application, we removed excessive gelatin from the exposed cortical surface with a flush of saline but left a covering layer in place. Muscimol was applied topically in four portions $(4 \mu \mathrm{l}$ each) producing drops covering the whole left auditory cortex. Diffusion time was limited to 2 min per drop with the residual liquid being removed afterward. Hence, the total effective muscimol dosage remains unclear, but, in all cases, the stimulus-evoked LFP signal (white noise stimuli, $60 \mathrm{db}$ SPL, $50 \mathrm{~ms}$ duration, rate of $1 \mathrm{~Hz}$, measured $30 \mathrm{~min}$ after muscimol application; one example is given in Fig. 12B) indicated a profound inactivation of the auditory cortex comparable with Talwar et al. (2001).

\section{Results}

Data were collected from a total of 192 units in 24 anesthetized Mongolian gerbils (oddball and roving oddball paradigm) targeting the vMGB with a multichannel recording approach (Fig. 2 B). Almost all of the recorded neurons (>95\%) showed a phasic onset response to the pure tone stimuli with a shortlatency burst of a few spikes (Figs. $1 A, 2 C$, top panel). We therefore restricted our analysis in most parts of this study to the first $30 \mathrm{~ms}$ of the stimulus-evoked response encompassing this onset component.

\section{Probability-dependent differences in neuronal response activity}

In a first series of experiments, we investigated SSA in 100 units (15 animals) by comparing neuronal responses to pure tones of different frequencies in an oddball paradigm. An example of a well isolated unit in the vMGB with differential responses to pure tones depending on stimulus probability is illustrated in Figure 1. Responses to the low-probability deviant tones $(p=0.1)$ that were presented among high-probability standard tones $(p=0.9)$ one octave apart were on average $17.6 \%$ (SI of 0.08 ) higher. In this example, the difference in response activity was independent of the frequency relationship (i.e., the deviant frequency being either higher or lower; configurations B1, B2) and was readily visible in single-trial comparisons between standard and deviant trials, as indicated in Figure $1 A$. The response of the unit was clearly phasic with a short latency after tone onset. The numbers of spikes per trial were small (one to four), but consistently different between standard and deviant trials (Fig. $1 A$, numbers on the right). Averaging the response of the unit over 1000 trials revealed a marked difference in the response distribution (spikes/ trial) between standard and deviant stimuli (Fig. $1 B$ ), again, for both test blocks (B1 and B2 in Fig. $1 B$, top and bottom, respectively). Comparing, for instance, the response distribution for $f_{1}$ [lower frequency (LF)] as the standard tone (Fig. $1 B$, top left histogram) with responses to $f_{1}$ as the deviant tone (Fig. $1 B$, bottom left histogram) reveals a clear shift from lower to higher spike rates. This is also true for the corresponding histograms of responses to the higher frequency (HF) $f_{2}$ (Fig. $1 B$, bottom and top right histogram).

SSA in the vMGB was quantified by assessing probabilitydependent changes in response rate. The median spike response rate (DS difference, deviant/standard) to the rare deviant stimuli was $15.4 \%$ higher (SI of 0.07 ) compared with responses to the highly probable standard stimuli ( 1 octave frequency separation, 0.1 deviant probability, repetition rate of $2 \mathrm{~Hz}$ ) (Fig. $2 \mathrm{~A}$ ). This was significantly different from the control condition with equal probability (50\%) for both tone frequencies (two-sample Kolmogorov-Smirnov test, DS/50\%, $p<0.001$ ). As expected, the average percentage change in the control condition was $\sim 0$ $(-0.02 \%$, median $)$. We further tested the possibility of a systematic asymmetry of neuronal responses in the stimulation pairs (relative lower vs higher frequency). Therefore, we split up the frequency pairs and calculated the relative change in response rate separately for each relative LF and HF (Fig. 2A). Average changes in response in both cases resembled the general "unit" effect (LF/50\%, $p<0.001 ; \mathrm{HF} / 50 \%, p<0.001)$. Although the single frequency distributions had a larger range, there was no statistical difference at the population level, neither in relation to the unit distribution (unit/LF, $p=0.56$; unit/HF, $p=0.44$ ) nor for direct comparison ( $\mathrm{LF} / \mathrm{HF}, p=0.96)$.

The time course of SSA during the typical neuronal response pattern to pure tones in the oddball paradigm was investigated in a time window of $60 \mathrm{~ms}$ covering the phasic onset response (Fig. 2C). Probability-dependent differences in spike rate could be found along the whole time course of the stimulusevoked response, consistently showing the pattern of higher activity in deviant-related responses. Absolute differences were larger around the peak of the onset response (Fig. 2C, middle). However, the absolute difference values are correlated with the absolute spike rate response values. Therefore, we calculated the change in percentage and normalized the scale effect by the nonlinear arcsin transformation. This arcsin-corrected distribution (Fig. 2C, bottom) indicated a difference of constant magnitude after response beginning for the whole time range investigated.

\section{Habituation of standard-related responses underlies stimulus-specific adaptation}

The probability-dependent difference in response activity as it was shown for the vMGB (see above) could, in principle, be caused by an increase in deviant-related activity, a reduced standard-related response, or some combination of both processes. To disentangle these processes possibly underlying SSA, we compared the neuronal responses measured during the oddball paradigm with activity measured for determining the response properties of the neurons (frequency response area and frequency response curve). Although these recordings had a different purpose at first, they included in most cases the two chosen 


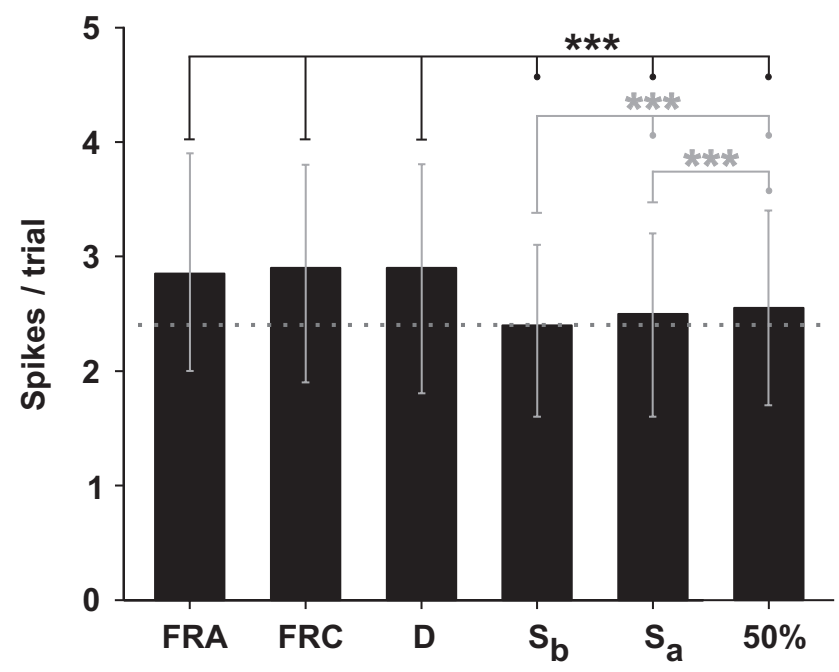

Figure 3. Comparison of probability-dependent activity levels between various stimulation paradigms $(n=73)$. Shown are average responses (population median \pm interquartile range of the averaged number of spikes per trial) to stimuli of different probability, derived from different stimulation paradigms applied. Comparison was done only for frequencies at $60 \mathrm{~dB}$ SPL that were tested in all of the following stimulus configurations: the FRA, the FRC, and four different stimulus types taken from the oddball paradigm. These included the deviant (D), the standard tone right before the deviant $\left(S_{b}\right)$, the standard tone right after the deviant $\left(S_{a}\right)$, and the tonal response in the control condition with equal probability for standard and deviant $(50 \%)$. Dotted line denotes the median of $S_{b}$. Statistical comparisons are indicated above the bars, with the asterisks representing the level of significance $\left.{ }^{* * *} p<0.001\right)$. Tests based on the two-sample Kolmogorov-Smirnov test are drawn in black, and those based on the Wilcoxon's signed rank test are colored in gray.

stimulation frequencies of the oddball paradigm as part of their stimulation scheme. We therefore analyzed all units with an exact match of tested frequencies at $60 \mathrm{~dB}$ SPL in all paradigms $(n=$ 73) (Fig. 3).

Comparably high levels of activity were elicited when tones of a given frequency occurred with low probability among several other tones, as was the case for determining the FRA (average probability of occurrence for a given frequency, $p=0.04$ ) and the FRC (average probability of occurrence for a given frequency, $p=0.04$ ). There was no statistical difference between spike activities evoked by deviants in the oddball paradigm and activities evoked by the same stimulus presented in the context of the frequency response area or the frequency response curve (twosample Kolmogorov-Smirnov test, FRA/deviant, $p=0.79$; FRC/ deviant, $p=0.79$; FRA/FRC, $p=0.99$ ) (Fig. 3). In contrast, activity levels in the three low-probability stimulus paradigms were significantly higher by $\sim 18 \%$ compared with the neuronal responses to the standard tone before each deviant $\left(S_{b}\right)$, which was always the last in a continuous sequence of equal tones (between 2 and 13 tones; standard tone probability, $p=0.9$ ). There was no statistical difference in activity levels between the highprobability stimuli using the two-sample Kolmogorov-Smirnov test. Nevertheless, there are highly significant differences revealed by the Wilcoxon's signed rank test $(p<0.001)$, which is based on a pairwise comparison between standard- and deviant-related spike rate rather than on the accumulated frequency distribution. The activity related to the first standard stimulus presented after the deviant stimulus $\left(S_{a}\right.$, i.e., two tones after $S_{b}$ ) (Fig. 3) was significantly higher compared with the response to the standard stimuli presented right before the deviant stimulus $\left(\mathrm{S}_{\mathrm{b}}\right)$. This pattern is characteristic for neuronal habituation. The higher post-deviant-related standard activity can be considered as a con-
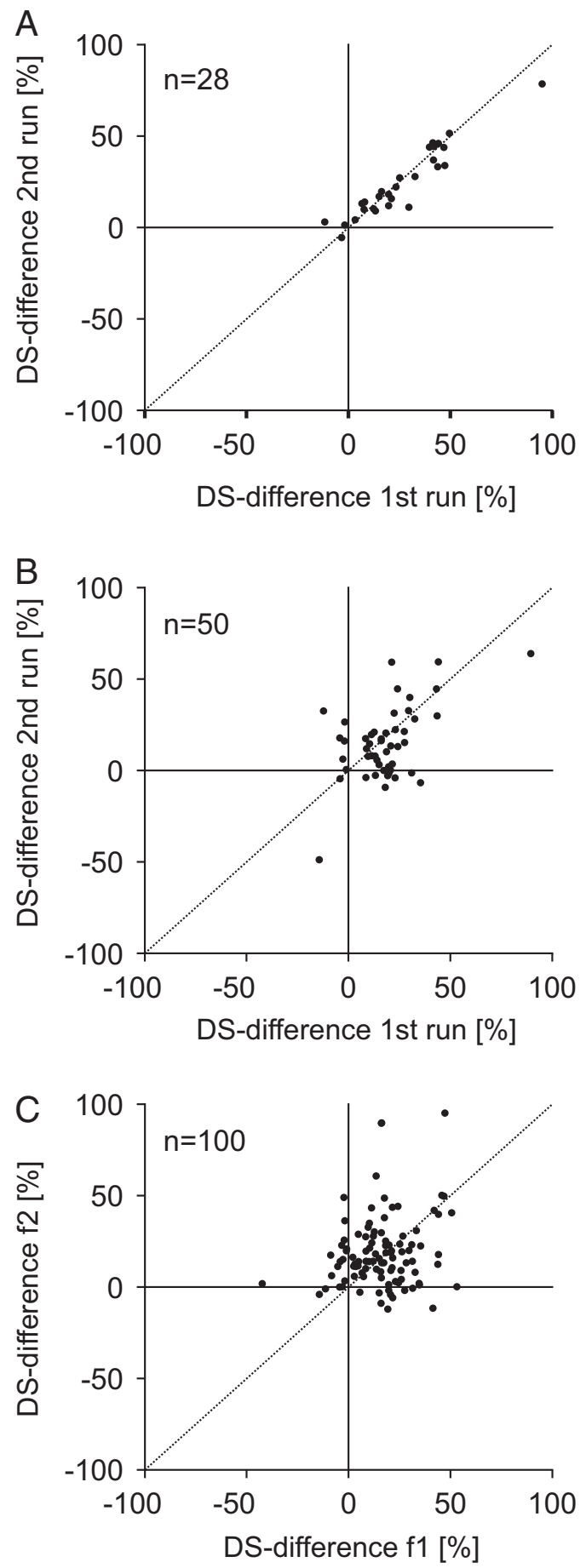

Figure 4. Frequency-specific effects of SSA in vMGB units. $A$, Comparison of DS-difference values (from 1st vs 2 nd stimulus set) taken from oddball paradigms repeated twice per unit. The complete stimulus set (10 blocks plus control conditions) was repeated immediately after the first presentation with the same frequency pair in 14 units (28 frequencies tested twice) to determine the stability of adaptation over time during the same recording experiment. Data points cluster along the diagonal (bottom left to the top right corner), indicating equal values for the repeated stimulus set. $\boldsymbol{B}$, Comparison of DS-difference values (from 1st vs 2nd stimulus set) taken from blocks of the oddball paradigm repeated with a slightly different frequency pair ( 0.4 octaves on average) in the same unit. Data from 25 units ( 50 frequencies for each stimulus set) are included. Data points scatter around the diagonal, indicating that adaptation is not independent of stimulation frequency. $\boldsymbol{C}$, Comparison of DS-difference values taken from the relative lower $\left(f_{1}\right)$ versus the relative higher $\left(f_{2}\right)$ frequency of each frequency pair tested in the oddball paradigm (100 units). Population data were already shown in Figure $2 A$. Once again, the data do not cluster along the diagonal. 
sequence of "dishabituation" caused by the presentation of a deviant tone between. Both types of standard-related activity were significantly reduced compared with the elicited response in the equal probability condition, in which responses were increased by $\sim 5 \%$ compared with the most habituated activity $\left(\mathrm{S}_{\mathrm{b}}\right)$. Therefore, we consider habituation to the highly probable standard as the main process underlying SSA.

\section{Is stimulus-specific adaptation frequency-specific?}

Although SSA is mostly seen as a neuronspecific property in the literature, there is still the possibility that the underlying habituating effects might differ within a frequency response area in vMGB neurons. This leads directly to frequency-specific adaptation, because tone frequency is the primary stimulus feature determining the response area.

One possible approach to this question is to repeat, for a given set of units, the recording stimulus set of the oddball paradigm with different frequency pairs. This, however, relies on stable recording and stable adaptation effects over time. We, therefore, determined the stability of adaptation effects in the recordings by comparing adaptation in recorded stimulus sets with identical stimulation frequencies that were acquired successively for a given unit. Disparities in the repeated recording should become obvious by plotting the DS-difference values of the first stimulus set against the DS difference of the second repeated stimulus set. DSdifference values from such comparisons, determined separately for the higher and lower frequency in each frequency pair, correlated strongly (Spearman's correlation coefficient, $r=0.95$ ) (Fig. 4A) and were very similar between the first and the second stimulus set (i.e., data points clustered along the diagonal). This indicated that the data acquired on adaptation were stable and highly reproducible.

A first approach to frequency-specific effects was then taken by shifting the stimulus pair in frequency (by 0.4 octaves on average) while maintaining the frequency separation between deviant and standard constant, giving rise to a second oddball stimulus set. Related DS-difference values to the first and second stimulus set were correlated $(r=0.53$ ) (Fig. $4 B$ ) but were much more scattered around the diagonal line of equality compared with repetitions with identical frequency pairs (Fig. 4A). Their scattered appearance indicates a marked frequency-specific difference in habituation. Finally, the most obvious frequencyspecific effects of adaptation were revealed by directly comparing DS differences between the two stimulus frequencies $\left(f_{1}, f_{2}, 1\right.$ octave apart) of each test pair that were used as corresponding deviant/standard combinations in the oddball paradigm (Fig. $4 C$ ). The related DS differences of $f_{1}$ versus $f_{2}$ from 100 units (15 experiments) had a broad distribution of correlated values that were clearly different in many cases. The majority of correlated data points were broadly dispersed from the diagonal $(r=0.20)$ (Fig. 4C). Furthermore, no significant correlation of the DSdifference magnitude with the spike rate was found (Spearman's correlation coefficient, $r=0.04, p=0.53$ ) excluding that scale effects account for the revealed pattern.

One example of how such frequency-specific differences in habituation could, in addition, be arranged inside a given frequency response area of a unit is shown in Figure 5. Testing several frequency pairs in an oddball paradigm revealed in this unit a systematic arrangement of DS differences over a frequency range of $\sim 1.5$ octaves. For all tested frequencies in the frequency response range at $60 \mathrm{~dB}$ SPL, DS differences were larger at the high-frequency slope $(34.2$ and $30.5 \mathrm{kHz}$ ) compared with DS differences at the more centrally located lower test frequencies (24.1 to $12.1 \mathrm{kHz}$ ).

A systematic variation of adaptation within a given frequency response range was also found in simultaneous recordings from several spatially separated units. These simultaneous recordings could exhibit either response areas with closely overlapping (Fig. 6) or clearly separated (Fig. 7) frequency ranges, both together providing a first indication for a possible topographic arrangement of adaptation effects. Recordings from three different units 
A
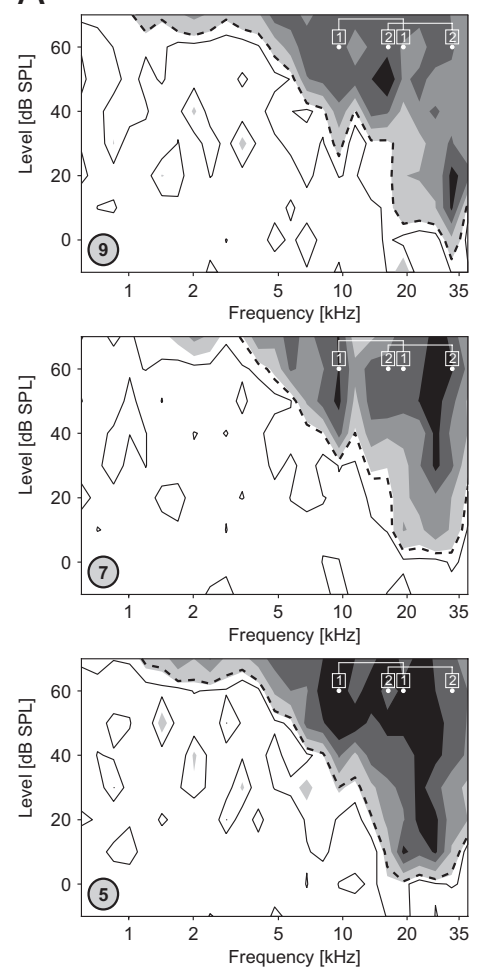
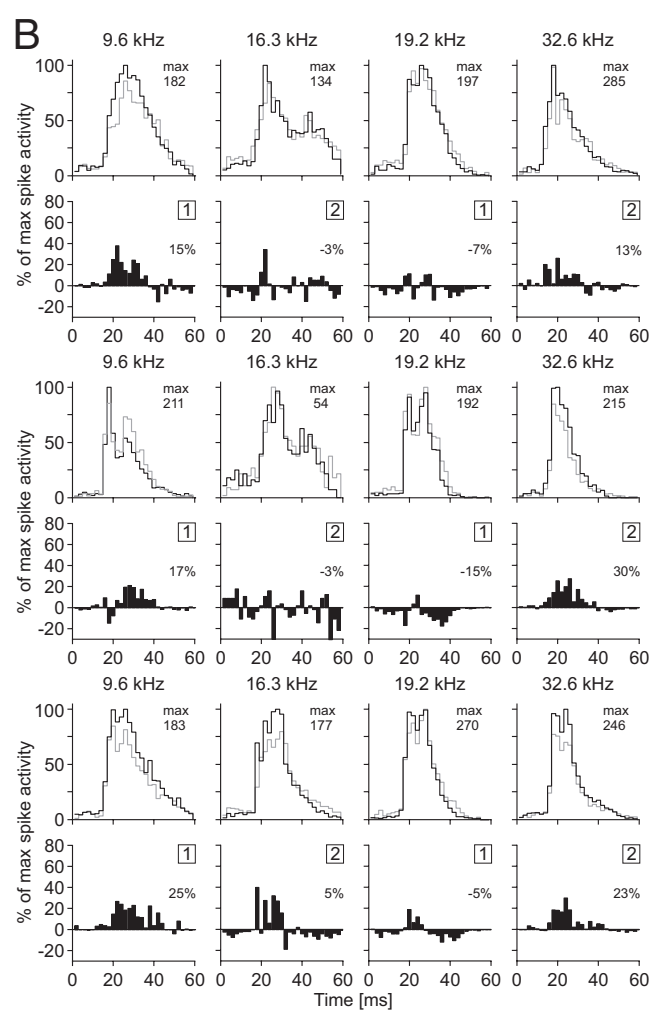

Figure 6. Example of frequency-specific differences in adaptation in three vMGB units recorded simultaneously from three different recording channels. The units were tested consecutively with stimulus sets of the oddball protocol using two different frequency pairs. $\boldsymbol{A}$, Frequency response areas of the three units. The tuning curve in each plot is marked by the dashed black line. White points at $60 \mathrm{dBSPL}$ indicate the tested frequencies, and the white numbers identify the two different frequency pairs in their chronological order. The number in the gray circle (bottom left corner) represents the recording channel. Areas enclosed in solid black lines exhibit an activity level exceeding the mean plus 1SD of the interstimulus activity level. Activity encoded in grayscale as indicated in Figure 5. $\boldsymbol{B}$, Plots to the right of each frequency response area present data for each respective unit. Shown are the average normalized standard-related (gray line) and deviant-related (black line) activity for the four different test frequencies (top row) and the difference in activity (deviant - standard; bottom row) depicted in PSTHs ( 2 ms bin width). The pure tone frequency is indicated above each column. The number of the stimulation pair for this frequency is given in the top right corner of each difference PSTH in the bottom row, with the percentage DS-difference value stated beneath.

that are spatially separated by at least $100 \mu \mathrm{m}$, but have very similar frequency response areas, are depicted in Figure 6. As already shown in Figures $4 C$ and 5, adaptation (i.e., DSdifference values) varied inside the frequency response areas. DS differences at the two frequencies located closer to the edges of the frequency response range $(9.6$ and $32.6 \mathrm{kHz})$ show larger difference values than those located in the central part (16.3 and $19.2 \mathrm{kHz}$ ). In addition, an astonishingly similar pattern of DS differences depending on frequency is obvious between these units. At $19.2 \mathrm{kHz}$, for example, a late component at $\sim 40 \mathrm{~ms}$ is visible across the three channels in which the standard elicited higher spike sums than the deviant.

Simultaneous recordings from units with response areas located in different frequency ranges showed frequency-specific differences in habituation as well (Fig. 7). Regions with low DS differences and regions with larger DS differences were systematically arranged along the frequency response curve, but with a different pattern for each unit. As a result, specific frequencies could cause in one unit a strong DS difference but had no adapting effect in another, simultaneously recorded unit. Hence, we conclude that habituation acts in a frequency-specific manner with an underlying topographic pattern. Moreover, the DS differences could not have been the effect of sensory adaptation or habituation at a peripheral level of the auditory pathway, such as the auditory nerve fibers. Instead, the measured habituation rather likely represents an effect generated in central parts of the auditory pathway.

\section{Time course of habituation}

It has been shown for cortical neurons that SSA takes place on several timescales (Ulanovsky et al., 2004). Therefore, we addressed this question for MGB neurons and analyzed the time course of adaptation in more detail. Specifically, we were interested in three questions. (1) Is the time course of this habituation a fast or a slow process? (2) Does the effect of habituation depend on the number of consecutive standard trials? (3) Is it the result of a nonlinear or continuous process?

The data recorded with the oddball paradigm were analyzed trial by trial to approach these questions (Fig. 8). The time course of activity for both deviant and standard responses over the whole (averaged) block of 1000 trials for all units was almost constant. However, as predicted by stimulus probability, the constant level of activity in deviant responses was clearly above the one in standard responses by $\sim 10$ percentage points of normalized activity (Fig. 8A). Although deviant responses seemed to be at the respective median level right from the first deviant stimulus, the median level of standard responses was reached only after several standard stimulus presentations. A visual inspection of this pattern at higher temporal resolution (Fig. 8B) revealed that the average level of standard activity was slightly above the deviant level right at the beginning and dropped sharply, reaching steady state somewhere between 50 and 100 trials. Furthermore, it became clear that deviant-related activity declined at the beginning as well, so that the time course of activity for both the standard as well as the deviant stimuli could be fitted with a decaying exponential of the type $f=c_{1}+c_{2}$ $* e^{(-t / \tau)}$, with $c_{1}$ being the asymptote, $c_{2}$ the decay factor, and $\tau$ the time constant. This suggests a common process of modulation with putative different effect sizes for both deviant and standard responses. Surprisingly, however, both exponential functions shared similar time constants of $\tau=20.44$ trials (deviant) and $\tau=20.35$ trials (standard) and hold the grand median difference of 10 percentage points already in their asymptotic terms (deviant, $c_{1}=67.8, c_{2}=10.4$; standard, $c_{1}=57.2, c_{2}=$ 11.5).

This led to the assumption that differences in the level of activity between standard and deviant might be induced at the very beginning of stimulation. To test this, a more detailed analysis of the temporal development of activity in three different temporal sections was performed (Fig. 8C). Activity in the three sections was analyzed using linear fits $\left(y=c_{1}+c_{2}{ }^{*} x\right)$. The left panel (trials 1-5) shows only standard stimuli as the first deviant was presented in trial 8. A strong decrement of 9 percentage points of standard activity occurred within the first three consec- 
utive standard stimuli and an additional decrement of 1 percentage point in the following two stimuli of the initial stage. This resulted in a total decrement of 10 percentage points in standard activity during the initial five consecutive standard trials, which equals the final difference in grand median activity level between standard and deviant stimuli. The time course of activity after presenting the first three standard trials is characterized by an additional but weaker decrement of spike activity within approximately the next 100 trials (trials 3-107), with a slope for standard activity of -0.072 percentage points per trial compared to a slope of -4.5 percentage points per trial for the first linear fit in the left panel. Furthermore, the time course of activity for the standard and the deviant tones in this second temporal section (trials 6-107 in the middle panel encompassing 11 deviant presentations) run almost in parallel. The deviant activity level not only shared the same overall dynamics (slope of -0.075 percentage points per trial) but also reflected the variability of the standard activity. This resulted in a high degree of correlation between deviant and standard activity levels (correlating median of deviant with the preceding standard tone, Spearman's $\rho, r=0.81, p=$ $0.0044)$. We conclude that the grand median disparity between standard and deviant activity was established by a very fast habituation of standard activity during the first stimulus presentations (Fig. $8 C$, left), followed by a stage of fatigue with a less pronounced decrease in activity affecting the standard as well as the deviant levels (Fig. 8C, middle). The dynamics finally leveled off in the third temporal section. Here, both standard and deviant activity reached a more or less constant level with a minimal tendency for recovery (slope: standard, 0.001 percentage points per trial; deviant, 0.0002 percentage points per trial). The correlation in temporal development between standard and deviant activity in the third section was significant $(r=0.24, p=0.024)$ but much weaker than in the second section.

The contribution of the initial fast habituating standard activity was further tested by splitting up the dataset with respect to the strength of the frequency-specific DS difference (Fig. $8 D$ ). There was a highly significant correlation of the degree of habituation for the initial five standard trials with the overall frequencyspecific DS difference $(r=0.25, p<0.001)$. The same pattern was also obvious for the second temporal section $(r=0.21, p<0.01)$. Interestingly, in the two categories showing $25 \%$ or more DS difference, activity in the second trial is already strongly habituated. Consequently, we also found a highly significant correlation of the degree of habituation with the overall frequencyspecific DS difference for the initial two standard trials $(r=0.35$, $p<0.001)$, exhibiting an even more pronounced correlation coefficient as it has been revealed for the complete first five trials. This fits nicely into the pattern of "fast habituation" described above. In all cases, variations were rather high because spike activity values in this analysis were based only on five tone presentations per trial number and tested frequency. Together, the important process underlying DS difference at the level of the vMGB seems to be a fast inhibition of standard responses that has a nonlinear dynamic and is already effective after the first trial.

\section{Stimulus history}

Short-term stimulus history was investigated by analyzing the level of deviant activity depending on the number of consecutive standard tones presented immediately before (Fig. $8 \mathrm{E}$ ). We tested the influence of short time stimulus history (measured in numbers of consecutive standard tones presented immediately before) on normalized deviant activity in a nonparametric ANOVA (Kruskal-Wallis test, df $=9, \chi^{2}=20.15, p=0.017$ ). This re-
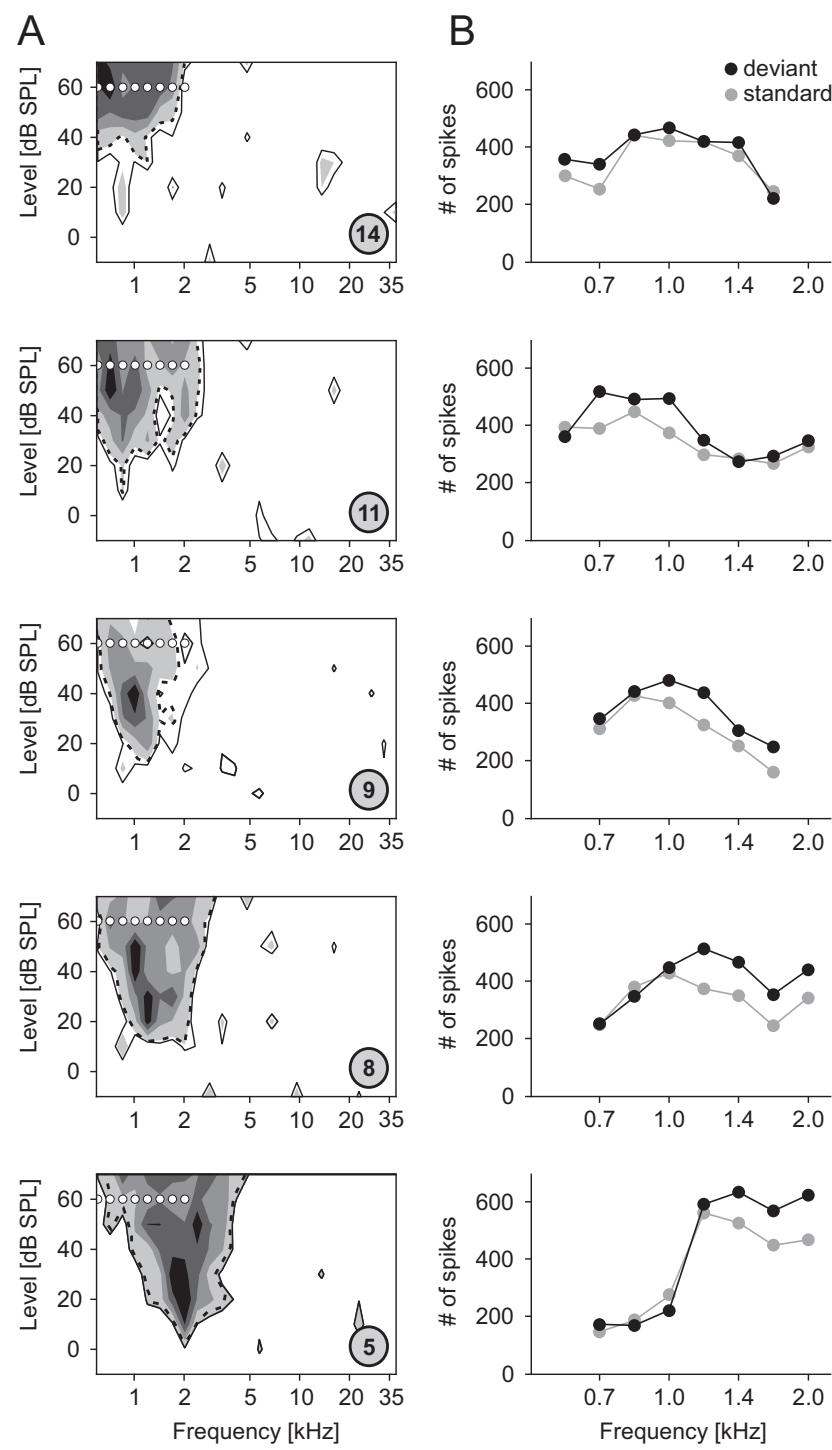

Figure 7. Example of frequency-specific differences in adaptation in five vMGB units recorded simultaneously from five different recording channels. The units were tested in a reduced oddball paradigm that allowed for testing an extended range of frequencies at the expense of a reduced number of standard/deviant comparisons (200 times). $\boldsymbol{A}$, Frequency response areas with the tuning curve in each plot marked by the dashed black line. White points at $60 \mathrm{~dB}$ SPL indicate the tested frequencies $(600,714,849,1009,1200,1427,1697$, and 2018 $\mathrm{Hz}$ ). The number in the gray circle (bottom right corner) identifies the recording channel. Areas enclosed in solid black lines exhibit an activity level exceeding the mean plus 1 SD of the interstimulus activity level. Activity encoded in grayscale as indicated in Figure 5. B, Plots to the right of each frequency response area show for each respective unit the absolute spike sum for standard (gray) and deviant (black) tones at the different test frequencies. Only data from frequencies inside the corresponding frequency response area are displayed.

vealed a small but significant increase in deviant activity level with an increasing number of preceding standard tones. The maximum range of this effect, however, was only 2.9 percentage points. Therefore, it was not a major factor influencing DS difference.

\section{Testing the fast habituation hypothesis with a roving oddball paradigm}

Frequency-specific effects of adaptation were so far only described in a limited frequency range and by using a lengthy paradigm. Within this paradigm, we could identify three stages of changes in spike rate behavior (fast habituation, fatigue, and steady stage). By taking into account the process of fast habitua- 

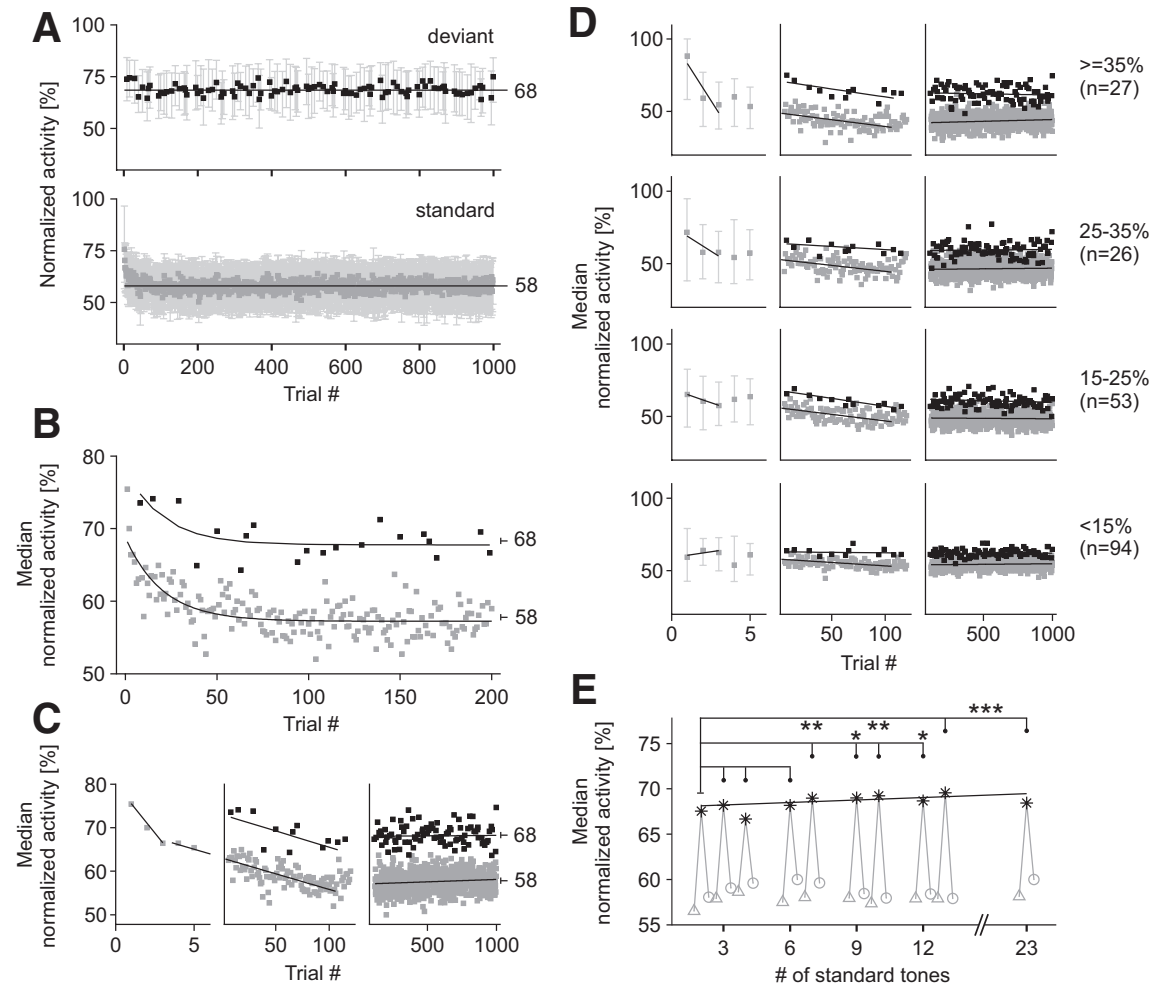

Figure 8. Time course of adaptation indicates that the fast habituating responses to standard stimuli predominantly determine DS differences. $A$, Average time course of normalized response activity from 100 units tested in the oddball paradigm (2 frequencies per unit). The plots show normalized spike activity, separated for deviant responses (top plot) and standard responses (bottom plot), averaged across the tested frequencies for all 1000 trials in a block ( $n=200$; median with interquartile range). Each data point included in the trial average includes itself the summed spikes from five repeated blocks, normalized to the maximal summed activity per trial for a given test frequency and unit. Black line denotes in both plots the grand median (value given as a number on the right). $\boldsymbol{B}$, Time course of response activity in a close-up view of the median values of the first 200 consecutive trials (black dots for deviant tones, gray for standard tones). Black lines denote exponential functions fitted to both time courses. Numbers and ticks on the right side indicate the level of grand median activity. C, Time course of standard (gray) and deviant (black) activity (only medians are shown for the 1000 trials), same as in $A$, but divided into three temporal sections (trials $1-5,6-120$, and 121-1000). Activities are fitted by linear functions $\left(y=c_{1}+c_{2}^{*} x\right)$, in the middle and right plot separately for standard and deviant stimuli. Numbers and ticks on the right side indicate the level of grand median activity. D, Same type of illustration as in C but dataset $(n=$ 200) split up into four different classes (organized in rows) according to the DS-difference values. Top numbers to the right indicate the DS-difference category and the bottom number in brackets the sample size. Activity in each temporal section is fitted by a linear function, in the middle and right plot separately for standard and deviant stimuli. $\boldsymbol{E}$, Median spike activity levels $(n=200)$ for deviant tones (marked as asterisks), the preceding standard tones (triangles), and the following standard tones (circles) as a function of the number of standard tones in between deviants. Thin gray lines connect the level of deviant responses with the preceding and following standard response. The deviant response after two standards (far left asterisk) was pairwise tested against the deviant responses with more standard tones between using a signed rank test. Asterisks in the top part of the plot indicate the level of significance $\left({ }^{*} p<0.05,{ }^{* *} p<0.01,{ }^{* * *} p<0.001\right)$. Significantly higher levels of activity in deviant responses occurred with increasing number of intermediate standard tones. The bold black line represents a linear fit to the deviant levels ( $y=0.064$ * (\# of standard tones) +68$)$.

tion within the first trials, we tried to determine DS differences on a shorter timescale and over a broad range of frequencies. Thus, we switched to the roving oddball design and tested 92 units in nine animals. In this paradigm, five consecutive tones of one frequency were followed by a frequency step (upward or downward) with a minimum of 1 octave, enabling us to test systematically a total frequency range of 2.5 octaves with 0.25 octave resolution. The first trial of a five-tone stimulus train was considered to be the deviant, the last trial (fifth) to be the standard. As shown for an example recording including three different units (Fig. 9), the roving oddball paradigm revealed broad frequency domains of marked DS difference. These regions covered different parts of the frequency response areas of simultaneously recorded units and were centered around different frequencies.
The average DS difference in this firstto-fifth tone comparison (Fig. 10 A), however, was small (median of 5.8\%) and broadly distributed (range from $-2.7 \%$ for the 25 th percentile to $15.1 \%$ for the 75th percentile). This effect was significantly smaller (two-sample KolmogorovSmirnov test, oddball/roving oddball, $p<$ 0.001) than the SSA observed in the oddball paradigm (median DS difference of $15.9 \%$, interquartile range from 5.6 to $25.7 \%$ ). The time course of activity in the five-tone series (Fig. 10B) resembled the one for the first five standard trials in the original oddball paradigm. Furthermore, comparable activity rates were obtained for the tested frequencies in the low-probability protocols of the tuning curve estimation and the determination of the frequency response curve (Fig. 10C). Compared with the deviant stimulus in the oddball design, however, the first tone of the five-tone series elicited a significantly lower spike activity level than the matching frequencies within the frequency response area (two-sample Kolmogorov-Smirnov test, FRA/first, $p<0.001)$ and the frequency response curve (FRC/first, $p=0.005)$. In fact, average spike rates to the first and the last tone (Fig. 10C, 1st, 5th) were so similar that the two-sample Kolmogorov-Smirnov test failed to pick up the difference $(p=0.36)$. Once again, there was a highly significant difference revealed by the Wilcoxon's signed rank test $(p<0.001)$. Therefore, we conclude that the two paradigms share in principle the same dynamics, but the activity in the roving oddball paradigm was probably reduced by a broad habituation attributable to the closely spaced test frequencies used here. We choose two ways to test this hypothesis. In a first approach, we compared the activities from first/fifth standard trials from the (standard) oddball paradigm as roving oddball substitutes and tested their activity difference against the first standard/deviant-tone combination (Fig. $10 D)$. Because the number of averages was low in this comparison, we gained values with a larger variation but comparable medians $(11.9 \%$ for first/fifth stimulus difference and $9.8 \%$ for seventh/eighth stimulus difference compared with $15.4 \%$ in the original population) and found no significant difference between both distributions (Wilcoxon's signed rank test, $p=0.78$ ). As a second approach, in three of the roving oddball experiments, we also recorded two frequency pairs with four blocks each of the oddball paradigm between the roving oddball stimulus set (Fig. 10E). As expected, we found higher DS differences within the oddball paradigm (median DS difference of $12.0 \%$ ) compared with the corresponding frequencies embedded in our roving oddball paradigm 
A
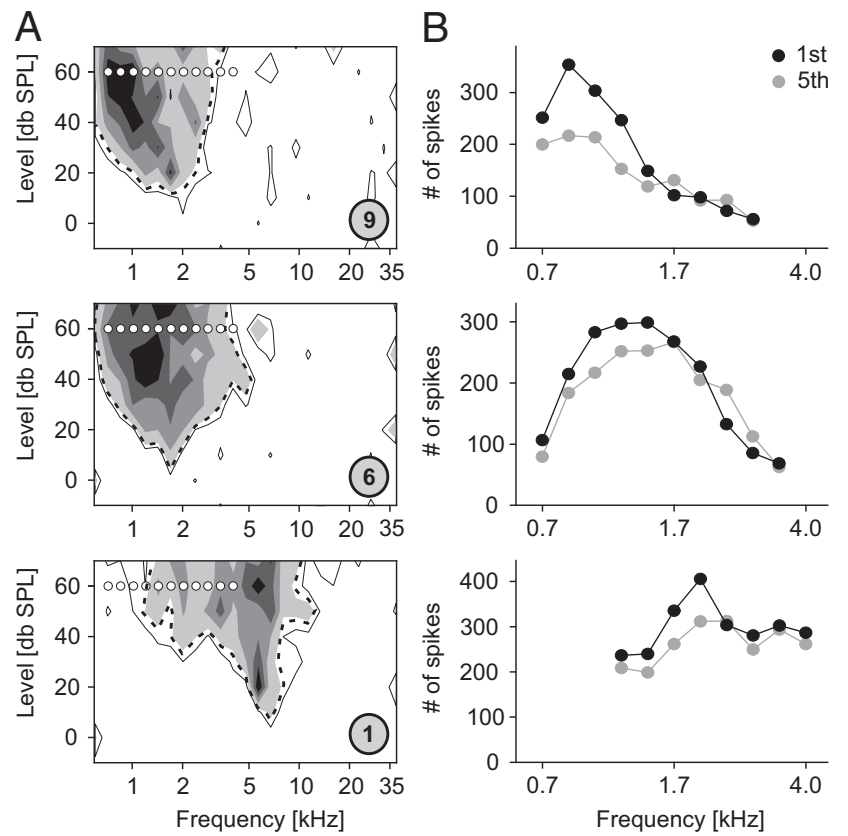

Figure 9. Example recording of DS differences determined with the roving oddball paradigm. $A$, Frequency response areas of three simultaneously recorded units. The level of activity encoded in grayscale as in Figure 5; recording channel numbers are indicated in the bottom right corners. Units were tested in a paradigm using 11 different frequencies $(714,849,1009,1200,1427,1697,2018,2400,2854,3394$, and $4036 \mathrm{~Hz})$. Frequencies are marked with white dots in the response plot at $60 \mathrm{~dB}$ SPL. B, Corresponding DS differences for each unit to the left noted as separate curves of spike sums for the first tone (deviant, black) and the fifth tone (standard, gray) within a five-tone stimulus train determined at different test frequencies. Only data from frequencies inside the corresponding frequency response area were analyzed.

(median DS difference of $4.3 \%$ ). Hence, we suggest that the same inhibitory mechanism was activated in both paradigms, resulting in an expanded and more general habituating interaction between stimulation frequencies that were clustered together in a narrow frequency band in our roving oddball design.

How adaptation effects were positioned in relation to the extent of the respective frequency response areas was investigated for 129 test frequencies (Fig. 10F), all of them in units without truncated flanks in the frequency range of $600-38,400 \mathrm{~Hz}$ at 60 dB SPL (i.e., with defined low- and high-frequency area borders). Absolute DS-difference values are shown as a function of the relative position in the response area along the frequency axis at $60 \mathrm{~dB}$ SPL. Positions were defined with respect to the closer frequency boundary (either upper or lower boundary). As depicted in Figure $10 \mathrm{~F}$, habituation was negatively correlated to the distance from the frequency boundary (Spearman's $\rho, r=-0.38$, $p<0.001$ ), demonstrating that, at the population level, effects located in the border region of the frequency response area show greater DS differences than those located in the central part. We tested the hypothesis of different effect strength by splitting up the dataset with respect to distance from the frequency boundary. The DS-difference distribution of frequencies located in the border region (distance $\leq 25 \%, n=30$ ) are significantly different from those in the central part (distance $>25 \%, n=99$; twosample Kolmogorov-Smirnov test, $p<0.001$ ). Some examples for pronounced effects in the border region of the frequency response area are given above (Figs. 5, 6). Nevertheless, there is a strong variation in DS differences at all positions, and, for a given unit, the relatively large DS differences could also be located in the central part of the frequency response curve. This was, for example, the case in some of the unit responses shown in Figure 7. For the population data (Fig. $10 \mathrm{~F}$ ), no additional correlations, e.g., to the steepness of the frequency response curve (Spearman's $\rho, r=0.017, \mathrm{NS})$ or to the relative $(r=0.093$, NS) or absolute $(r=$ $-0.074, \mathrm{NS})$ firing rate were found.

\section{Influence of cortical inactivation on adaptation in the vMGB}

One of the likely mechanisms for generating adaptation effects in the vMGB was tested in a final set of experiments: the well described adaptation at the cortical level (Ulanovsky et al., 2003, 2004) might influence the vMGB via direct or indirect descending projections. This was investigated for a subset of units (25 units from four animals), for which we successfully measured SSA in the vMGB with the roving oddball paradigm first under normal conditions (as above) and then while the ipsilateral auditory cortex was inactivated by a topical application of muscimol (Figs. 11, 12). However, with our approach, we were not able to determine a recovery afterward because of time limitations on the duration of stable recording conditions. Although clear DS differences were present under normal recording condition, they disappeared with cortical inactivation by muscimol as shown by the examples given in Figure $11 \mathrm{~B}$. This effect is also obvious at the population level. The distribution of DS-difference values before and after muscimol application (Fig. 12A) changed significantly (two-sample Kolmogorov-Smirnov test, $p<0.001$ ). Although for the control condition the distribution was significantly different from zero (Wilcoxon's test, $z=-4.13, p=0.000037$ ), thereby indicating a majority of positive DS-difference values, this was not the case anymore after muscimol application (Wilcoxon's test, $z=-1.16, p=0.25)$. In detail, the distribution of DS-difference values became symmetrical around zero by muscimol application (kurtosis of 3.9 and 0.9 for the recording without and with cortical inactivation, respectively). All these effects occurred in addition to the general decrease in stimulus-evoked spiking activity (Figs. $11 C, 12 C$ ) as a result of cortical inactivation, which accentuates the importance of auditory cortical activity for information processing in the vMGB. The general reduction in spike activity levels were observed for both the first and the fifth tone activity (Fig. 12C). Hence, we conclude that the auditory cortex plays a crucial role in the generation of SSA at the subcortical level of the auditory thalamus.

\section{Discussion}

SSA was investigated in the vMGB to determine frequencyspecific effects and their possible underlying mechanisms. It is an exciting feature of the auditory thalamus that the potential strength of SSA is anatomically segregated. So far, SSA in the vMGB as part of the lemniscal auditory pathway could not be detected at all (Ulanovsky et al., 2003, 2004) or showed SSA only to a relatively limited degree (Anderson et al., 2009; Yu et al., 2009; Antunes et al., 2010). Here, we present significant SSA in the gerbil vMGB with an average DS differences of $\sim 15 \%$, which is comparable with the $30 \%$ described for the rat MGB as a whole (Yu et al., 2009). These less pronounced effects in the rather sharply tuned vMGB neurons is consistent with results from the rat IC, in which tuning sharpness was inversely related to the amount of adaptation (Malmierca et al., 2009).

We used larger frequency differences in the oddball paradigms compared with the literature and tested over a much broader frequency range. This approach enabled us to systematically investigate frequency-specific effects. We also explored the possible 

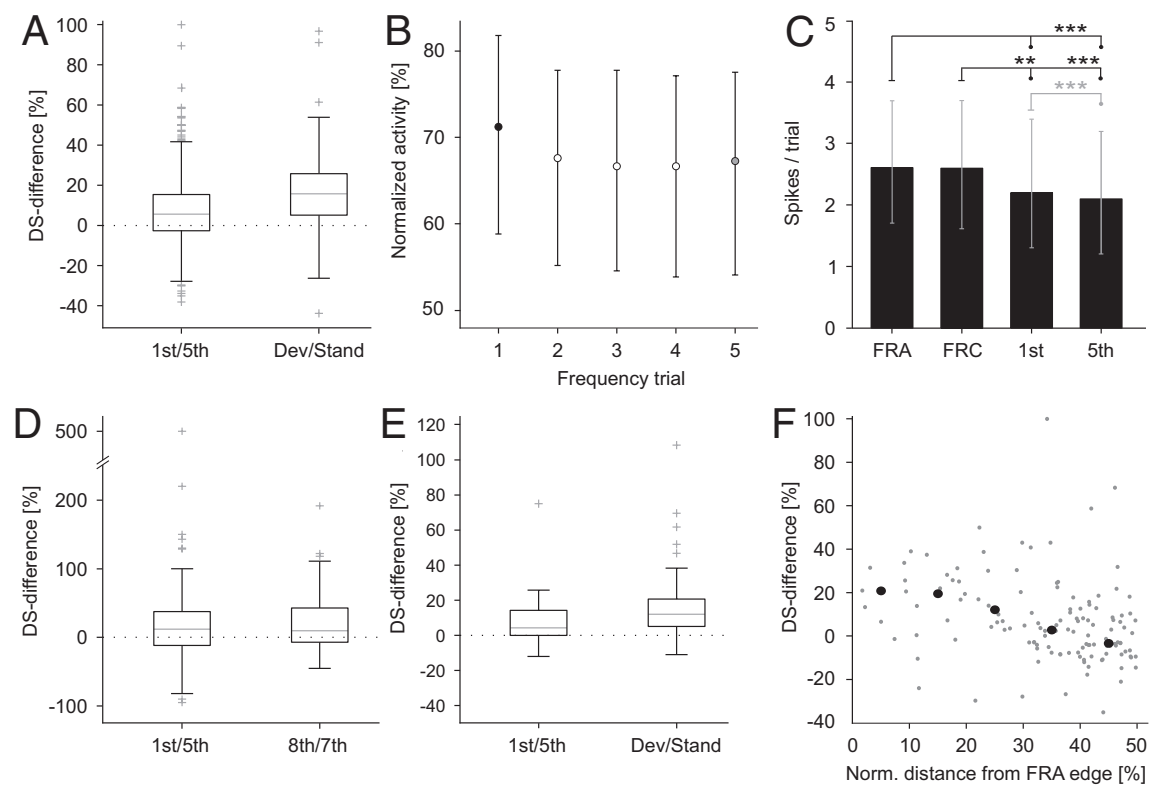

Figure 10. Population analysis for data recorded in the roving oddball paradigm. $\boldsymbol{A}$, Average DS difference (1st/5th) determined in the roving oddball paradigm ( $n=532$ frequencies in 92 units). Data were calculated by comparing responses to the first tone (deviant) with responses to the fifth tone (standard) of the five-tone stimulus train. Data from the oddball paradigm, already given in Figure 2, were redrawn to the right for comparison. $\boldsymbol{B}$, Time course of response activity to the five consecutive tones in the five-tone stimulus train normalized to the frequency-specific maximum spike sum per trial of a given unit (median with interquartile range). The first tone (deviant, black) and the fifth tone (standard, gray) are used to calculate DS differences. C, Comparison of activity levels (median \pm interquartile range of the number of spikes per trial) at specific frequencies ( $60 \mathrm{~dB} \mathrm{SPL}$ ), tested in all of the following stimulus configurations: in the FRA, the $F R C$, and the roving oddball paradigm (1st and 5 th tone of the five-tone stimulus train). Tests based on the two-sample Kolmogorov-Smirnov test are drawn in black, and those based on the Wilcoxon's signed rank test are colored in gray. Significant differences are indicated at the top with the level of significance encoded as ${ }^{* *} p<0.01,{ }^{* *} p<0.001$. D , Population data recorded in the oddball paradigm comparable with the roving oddball effects ( $n=100$, as shown in Fig. 2). Left side (1st/5th): Trial-specific differences in activity (comparable with DS difference) between the first standard tone and the fifth standard tone (roving oddball substitute). Right side (8th/7th): Trial-specific differences in activity between the first deviant (trial 8) and the preceding standard tone (trial 7). Data for relative low and high frequency $\left(f_{1}, f_{2}\right)$ are combined per unit to compensate for the small number of repetitions ( 5 per frequency and unit). $\boldsymbol{E}$, Direct comparison of the DS differences in the oddball paradigm (left, 200 single trials per given frequency in the standard and the deviant position) and the roving oddball paradigm (right, 240 single trials per given frequency for the 1 st and 5 th position) recorded in the same set of neurons at corresponding frequencies $(n=52)$. Frequency data were included, if both frequencies of a stimulation pair within the oddball paradigm were located inside the tuning curve and fulfilled the stability criteria (see Material and Methods). $\boldsymbol{F}$, Dependence of DS difference on the relative frequency position at $60 \mathrm{~dB}$ SPL in vMGB units. The positions of test frequencies were determined as relative positions in the frequency response area between the center $(50 \%)$ and the closer frequency boundaries (0\%; either top or bottom one) of the frequency response area for 129 frequencies recorded from 22 units. Depicted are normalized absolute DS differences (1st vs 5 th tone in the roving oddball paradigm) as a function of the relative frequency position (gray dots). The median of all data points within a $10 \%$ distance step are shown in black.

involvement of cortical feedback in SSA, which has been a matter of considerable debate in the literature. Here we demonstrate for the first time that the cortex indeed plays a crucial role.

\section{SSA is frequency-specific}

The stimulus feature mainly used in the literature to investigate SSA is a deviance in the tone frequency with the gained effects usually being described in a cell-specific manner. Although single neurons are definitely important units of information processing, differential responses to distinct stimuli cannot be the result of a mechanism acting at the level of the output mechanism of the cells, i.e., at the level of the soma. Therefore, it is most likely that SSA depends on specific input processing presumably located at different branches of the dendritic tree (Ulanovsky et al., 2004). Consequently, together with the dependency on the input channel, one can also expect dependency on the input parameters.
We tested such dependence and found a marked influence of stimulus frequency within the response range of the neurons, such that some frequency ranges showed strong and other only weak levels of SSA (Fig. 5). These ranges were similar for simultaneously recorded units with comparable frequency response areas and different for those with varying frequency response areas (Figs. 6, 7). Higher levels of SSA were, in addition, on average associated with the border regions of the frequency response range, which might reflect the characteristic of inhibitory projections.

Such frequency-specific effects were already obvious when comparing frequencydependent indices, because they markedly deviated from the diagonal (Fig. 4). Although comparable patterns with Figure $4 C$ were published several times, the obvious frequency-specific effects were not interpreted as such (Ulanovsky et al., 2003; Malmierca et al., 2009; von der Behrens et al., 2009; Yu et al., 2009). Testing neurons for SSA with paradigms including several frequencies simultaneously, as it was shown here, would probably reveal that most if not all cells in the IC, MGB, or the auditory cortex exhibit SSA in a certain frequency range.

It has been shown in the literature that the magnitude of SSA further depends on a variety of stimulus parameters such as frequency separation, stimulus probability, and repetition rate. Perhaps in part because of differences in these parameters, some previous studies have reported rather strong effects (SI $<1$ : Ulanovsky et al., 2003 for the AC; Antunes et al., 2010 for the MGB), whereas others have found only small effects (SI < 0.5: von der Behrens et al., 2009 for the AC; Anderson et al., 2009 for the MGB). The SSA values gained here were also rather small, although the stimulus parameters applied should have been suitable for eliciting strong effects. We believe that cell selection plays a crucial role in determining the potential magnitude of SSA. By focusing on a defined subpopulation of neurons with narrow tuning curves, we could present data from a range of paradigms with, for example, differences in deviant probability, which were rather consistent. We further restricted the analysis of the standard response in our DS-difference measure to the last stimulus preceding the deviant, but calculating a contrast measurement involving an average over all standard stimuli gave almost identical effect magnitudes because the time course of habituation was so fast and the effect of short-term stimulus history was rather small.

\section{Time course underlying adapting firing rates}

Our detailed analysis of the time course revealed that the most likely mechanism underlying SSA is a fast habituation of the neuronal response to the high-probability standard tone within the first few trials. The habituating response to the standard stimulus 
determining the degree of SSA (Fig. 3) is comparable with most studies at other levels of the auditory pathway and with human EEG recordings (Haenschel et al., 2005). Although the decrease in response to standard stimuli is usually strong in the first part of a trial sequence (Ulanovsky et al., 2003, 2004; Malmierca et al., 2009), we found an astonishingly fast time course with on average $54 \%$ of the reduction in firing rate occurring in the second trial and an additional $36 \%$ in the third standard trial (Fig. 8). This timing was surprisingly similar to human EEG recordings (Garrido et al., 2009), indicating that this mechanism once again might be a common feature of SSA.

The fast habituation phase in the MGB is followed by a second phase in both standard- and deviant-related responses with very similar time constant indicating general "fatigue," which probably accounts for the similarities of the exponential fits in Figure 8 B. The steady-state level of adaptation is on average determined by deviant probability. This is indicated by the strong neuronal response to the deviant in the $10 \%$ oddball paradigm as well as in the frequency response area and the frequency response curve compared with the control condition (50\%) and the lower level of standard-related activity (Fig. 3). Ulanovsky et al. (2004) suggested that stimulus history operates on two independent timescales: a local stimulus history with a time constant of two trials and a global stimulus history adapting to the long-term stimulus metastatistic (overall stimulus probability). Our data (Fig. 8), however, suggest sequential interactions of habituating and dishabituating processes that already exist within the very first trials. These sequential interactions balance the activity level trial by trial, consistent with the illustrated SSA time course of the mean population response in cortical neurons (Ulanovsky et al., 2003). One correlate of such fine-tuned processes is the overall dependency of the deviant activity level on the number of preceding standard stimuli (Fig. $8 E$ ).
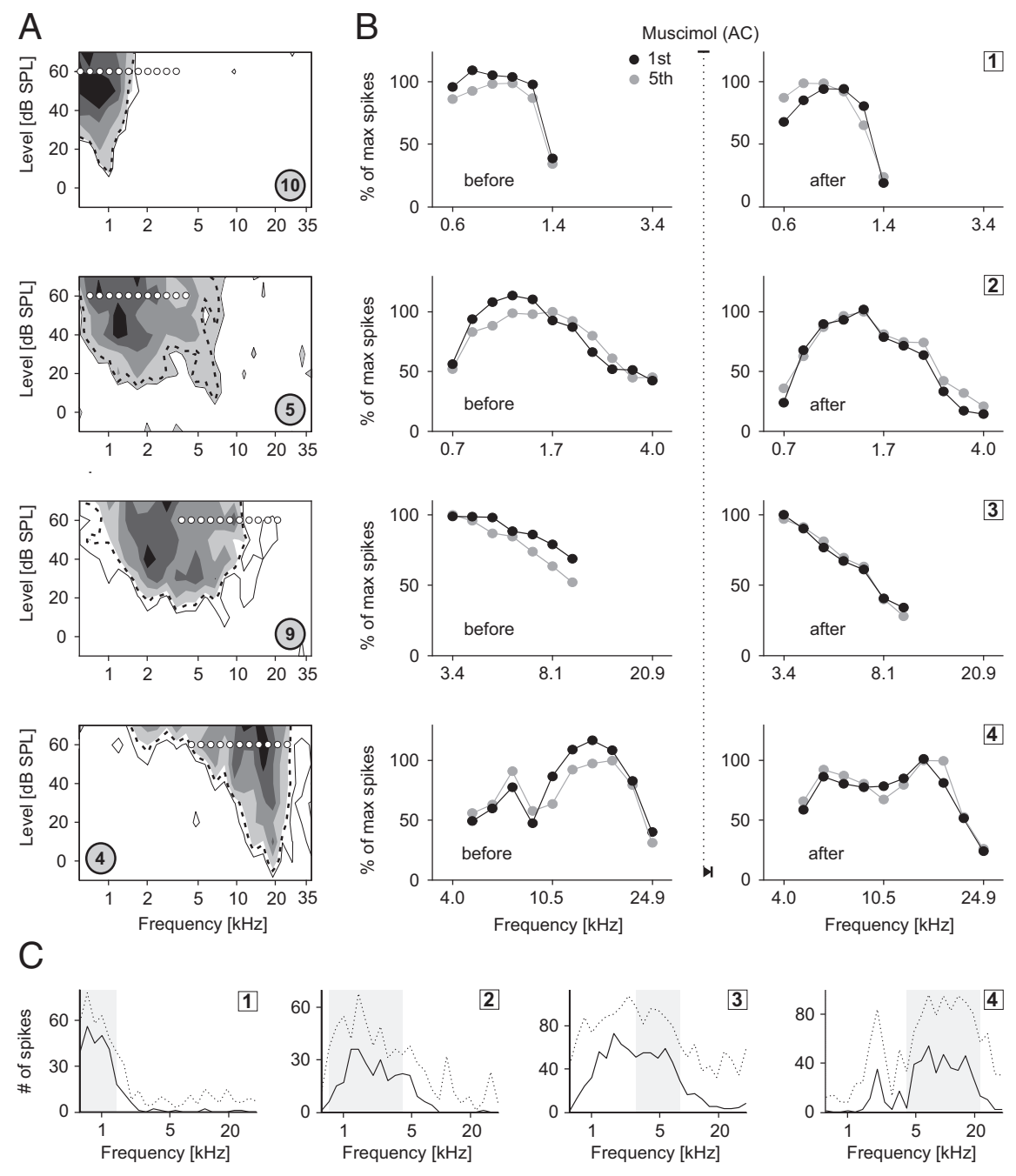

Figure 11. Roving oddball paradigm in the vMGB combined with cortical inactivation. $A$, Four examples of frequency response areas of vMGB units recorded in four different experiments (same type of illustration and annotations as in Fig. $9 A$ ) representing the whole frequency range covered. The level of activity is encoded in grayscale and additional information noted as in Figure 5. The roving oddball paradigm was performed using 11 frequencies (at $60 \mathrm{dBSPL}$ ) separated by 0.25 octaves in a range adjusted to the response range of the unit (top, 600-3394 Hz; top middle, $714-4036 \mathrm{~Hz}$; bottom middle, $3394-20,938 \mathrm{~Hz}$; bottom, $4036-24,900 \mathrm{~Hz}$ ). Position of test frequencies are marked with white dots in the response plot at $60 \mathrm{~dB}$ SPL. $\boldsymbol{B}$, Corresponding levels of activity depending on stimulation frequency for the first tone (deviant, black) and the fifth tone (standard, gray). Spike numbers were normalized to maximum spike sum activity elicited by the fifth tone within a given panel to compensate for the reduced activity level after muscimol application. Panels in the left column in $\boldsymbol{B}$ display normalized spike sums before application of muscimol to the auditory cortex (AC) and panels in the right column after cortical inactivation. Examples in each row are numbered as noted in the top right corner from top to bottom (boxed numbers). Only data from frequencies inside the corresponding frequency response area were analyzed. $\boldsymbol{C}$, Test recordings of frequency response curves from the four example units shown in $\boldsymbol{A}$ using 25 frequencies ranging from $600-38,400 \mathrm{~Hz}$ (spacing 0.25 octaves) and comparing the activity before (dotted line) and after (solid line) application of muscimol to the auditory cortex. Boxed numbers in the top right corner of each panel indicate the example number. The frequency range marked with light gray (background) indicates the range of stimulation inside the corresponding tuning curve.

\section{Roving oddball paradigm: tradeoff between frequency range and effect size}

We selected, based on the described fast habituation, another stimulation paradigm with additional power to reveal frequency-specific effects, the roving oddball paradigm (Garrido et al., 2009). Although this paradigm worked in principle, overall DS differences revealed were comparably small. The chosen narrow frequency spacing probably induced inhibitory/habituating side effects between the stimulation tones that resulted in a broadband adaptation of the whole frequency band used, thereby limiting the residual degree of variation. The influence of frequency separation on the degree of SSA is well documented in the literature, but in no case was this compared with corresponding spike levels from other paradigms.

\section{Corticofugal control of SSA in the auditory pathway}

Although on one hand, the strong effects of SSA described for the IC (Pérez-González et al., 2005; Malmierca et al., 2009) might simply be passed on to the MGB, inactivation of the TRN, on the other hand, directly eliminated SSA in the MGB (Yu et al., 2009). Because all three subcortical structures (IC, MGB, and TRN) are under auditory cortical control, the adaptation found there (Ulanovsky et al., 2003; von der Behrens et al., 2009) might very 
A

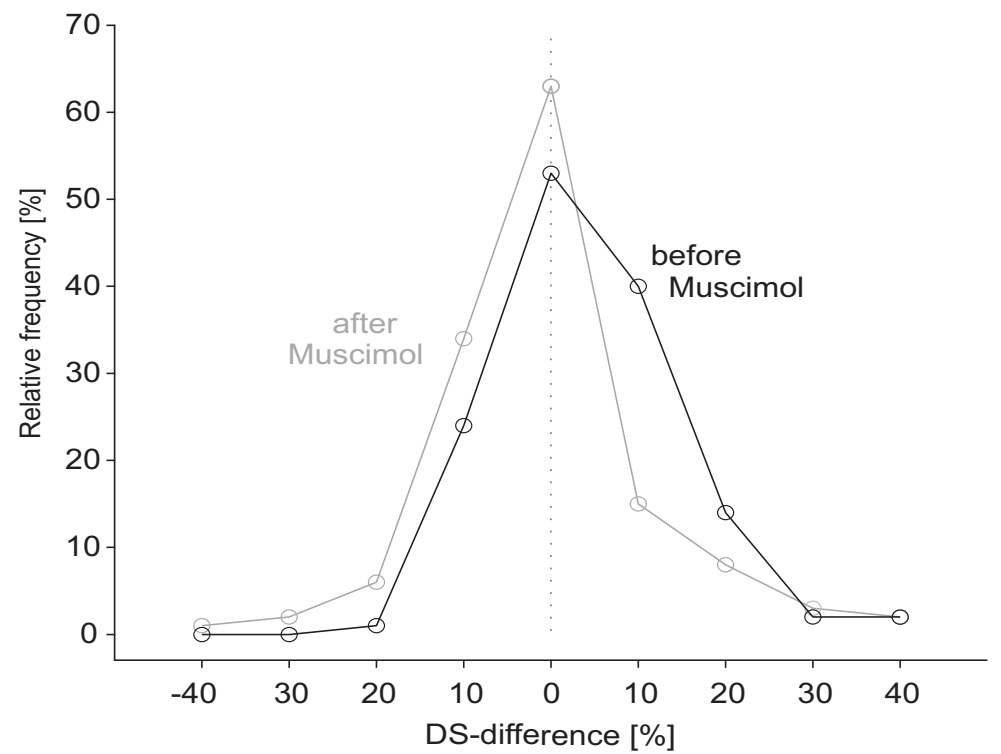

B

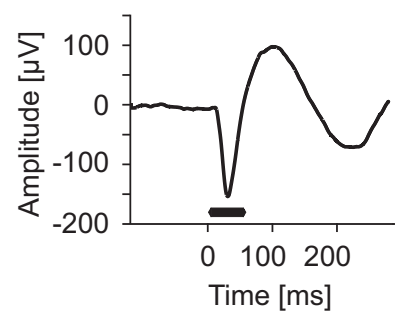

C

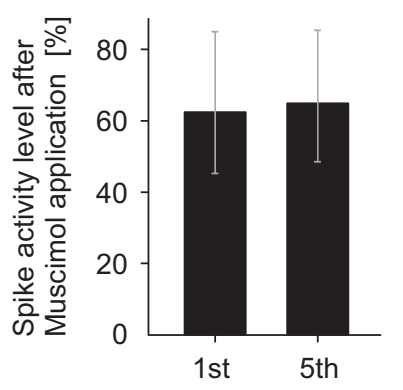

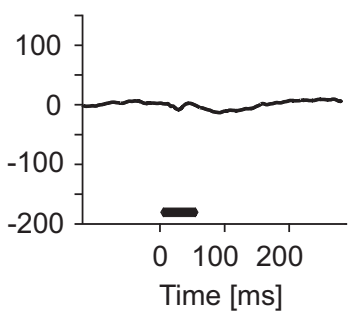

D

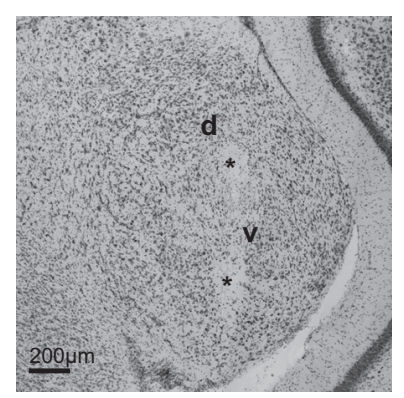

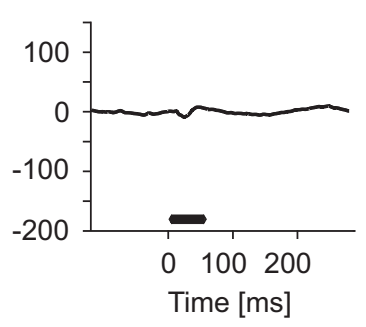

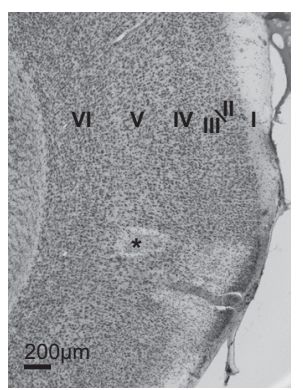

Figure 12. Cortical inactivation eliminates adaptation in the vMGB as indicated by the distribution of DS differences. $\boldsymbol{A}$, Distribution of determined DS differences in VMGB units ( $n=136$ frequencies in 25 units) before (black) and after (gray) cortical inactivation with muscimol (bin size, 10\%). $\boldsymbol{B}$, Inactivation of the auditory cortex was monitored in recorded LFPs. The example given here shows the LFP in layer V before muscimol application (left), 30 min after muscimol application (middle), and at the end of the experiment (right). Black horizontal bar indicates stimulus duration (white noise, $50 \mathrm{~dB} \mathrm{SPL}$ ) to determine LFP responses. $\boldsymbol{C}$, Spike activity levels (median \pm interquartile range) of the first and the fifth tone in the roving oddball paradigm after muscimol application as percentage values of the spike sum obtained before muscimol application. Muscimol caused an overall median decrease in activity levels of $35.8 \%$ in the vMGB. D. Examples of histological verification of electrode positions. Left, Coronal section through the MGB with lesions indicating the position of recording channels 1 and 16 (asterisk). Scale bar, $200 \mu \mathrm{m}$. v, Ventral part of the MGB; $d$, dorsal part of the MGB. Right, Lesion in layer $V$ of the auditory cortex indicating the electrode position for recording LFPs. Scale bar, $200 \mu \mathrm{m}$. Cortical layers are indicated by Roman numerals. One thalamic recording example corresponding to the histological data in $\boldsymbol{D}$ as well as the LFPs in $\boldsymbol{B}$ is given in Figure $11 A$ (bottom).

well lead to adaptive control of structures along the ascending auditory pathway. Thereby, the cortex could adjust its own input depending on the actual context of information processing. This mechanism might not provide a fast feedback onto the same stimulus processing (Anderson et al., 2009) but be effective in between-trial influences. Pharmacological inactivation of the auditory cortex using muscimol, meant as a simple test of this hypothesis, resulted in a clear reduction of SSA in the vMGB (Fig. 12).
Subcortical auditory processing is still active in the Mongolian gerbil, when the auditory cortex is blocked by muscimol (Goldschmidt et al., 2010; Happel et al., 2010). Conversely, $>5 \mathrm{~h}$ are necessary for reversal of cortical inactivation (Talwar et al., 2001; Happel et al., 2010), which exceeded our time of stable recording condition. Therefore, we are not able to present data for postinactivation recovery of thalamic SSA to definitively exclude unexpected side effects of muscimol treatment or direct diffusion of the drug into vMGB. Nevertheless, both possibilities are quite unlikely. Muscimol application was performed extremely carefully with a covering layer of gelatin in place functioning as a kind of sponge to prevent unintentional application to other brain areas. Direct diffusion for auditory cortex to vMGB would require that the drug would have to cross the cortical white matter, the hippocampus, and the ventricles finally to reach the MGB or alternatively to travel along the cortical white matter through the striatum and the anterior thalamic nuclei. In both cases, muscimol would not only have to spread a remarkable distance but would also affect large parts of the brain, including other important structures for auditory information processing (e.g., the IC via the ventricles or the TRN as part of the anterior thalamus), presumably resulting in different effects than those reported here.

Experimental approaches using inactivation with muscimol (Zhang and Suga, 1997; Yan and Suga, 1999; Zhang and Yan, 2008), cooling, and electrical stimulation (He, 1997; He et al., 2002; He, 2003b) of the auditory cortex demonstrated a general excitatory cortical influence on the MGB. Accordingly, cortical inactivation resulted in an overall decrease in firing rate of $35.8 \%$ (Fig. 12C). Conversely, stimulating the auditory cortex with a single electrical pulse can evoke inhibition (He et al., 2002). Speculating on such inhibitory mechanisms has to take into account the time constant of synaptic currents in the MGB of at most a few hundred milliseconds (Bartlett and Smith, 2002; Yu et al., 2004; Asari and Zador, 2009), which is too fast compared with our $500 \mathrm{~ms}$ interstimulus interval. Metabotropic receptors, however, such as the $\mathrm{GABA}_{\mathrm{B}}$ receptor with its potassium conductance have a longer time constant and are plausible candidates in the MGB (Tennigkeit et al., 1998, 1999; Schwarz et al., 2000). Because GABAergic interneurons are missing in the rodent vMGB (Winer and Larue, 1996; Arcelli et al., 1997), the cortex could act via the GABAergic projection neurons from the TRN (Crabtree, 1998; Winer et al., 1999) as demonstrated recently (Zhang et al., 2008). The axonal projection pattern of TRN cells as described in the rat (Kimura et al., 2007) is well suited to 
execute a tonotopically organized corticofugal gating function and could account for the pattern of SSA within and across the frequency response areas as shown above.

\section{References}

Abel C, Kössl M (2009) Sensitive response to low-frequency cochlear distortion products in the auditory midbrain. J Neurophysiol 101:1560-1574.

Allen TA, Narayanan NS, Kholodar-Smith DB, Zhao Y, Laubach M, Brown TH (2008) Imaging the spread of reversible brain inactivations using fluorescent muscimol. J Neurosci Methods 171:30-38.

Anderson LA, Christianson GB, Linden JF (2009) Stimulus-specific adaptation occurs in the auditory thalamus. J Neurosci 29:7359-7363.

Antunes FM, Covey E, Malmierca MS (2010) Is there stimulus-specific adaptation in the medial geniculate body of the rat? In: The neurophysiological bases of auditory perception (Lopez-Poveda EA, Palmer AR, Meddis R, eds), pp 535-544. New York: Springer.

Arcelli P, Frassoni C, Regondi MC, De Biasi S, Spreafico R (1997) GABAergic neurons in mammalian thalamus: a marker of thalamic complexity? Brain Res Bull 42:27-37.

Asari H, Zador AM (2009) Long-lasting context dependence constrains neural encoding models in rodent auditory cortex. J Neurophysiol 102:2638-2656.

Bartlett EL, Smith PH (2002) Effects of paired-pulse and repetitive stimulation on neurons in the rat medial geniculate body. Neuroscience 113:957-974.

Crabtree JW (1998) Organization in the auditory sector of the cat's thalamic reticular nucleus. J Comp Neurol 390:167-182.

Garrido MI, Kilner JM, Kiebel SJ, Stephan KE, Baldeweg T, Friston KJ (2009) Repetition suppression and plasticity in the human brain. Neuroimage 48:269-279.

Goldschmidt J, Wanger T, Engelhorn A, Friedrich H, Happel M, Ilango A, Engelmann M, Stuermer IW, Ohl FW, Scheich H (2010) Highresolution mapping of neuronal activity using the lipophilic thallium chelate complex TIDDC: protocol and validation of the method. Neuroimage 49:303-315.

Haenschel C, Vernon DJ, Dwivedi P, Gruzelier JH, Baldeweg T (2005) Event-related brain potential correlates of human auditory sensory memory-trace formation. J Neurosci 25:10494-10501.

Happel MF, Jeschke M, Ohl FW (2010) Spectral integration in primary auditory cortex attributable to temporally precise convergence of thalamocortical and intracortical input. J Neurosci 30:11114-11127.

He J (1997) Modulatory effects of regional cortical activation on the onset responses of the cat medial geniculate neurons. J Neurophysiol 77:896-908.

He J (2003a) Corticofugal modulation of the auditory thalamus. Exp Brain Res 153:579-590.

He J (2003b) Corticofugal modulation on both ON and OFF responses in the nonlemniscal auditory thalamus of the guinea pig. J Neurophysiol $89: 367-381$

He J, Yu YQ, Xiong Y, Hashikawa T, Chan YS (2002) Modulatory effect of cortical activation on the lemniscal auditory thalamus of the guinea pig. J Neurophysiol 88:1040-1050.

Kimura A, Imbe H, Donishi T, Tamai Y (2007) Axonal projections of single auditory neurons in the thalamic reticular nucleus: implications for tonotopy-related gating function and cross-modal modulation. Eur J Neurosci 26:3524-3535.

Malmierca MS, Cristaudo S, Pérez-González D, Covey E (2009) Stimulusspecific adaptation in the inferior colliculus of the anesthetized rat. J Neurosci 29:5483-5493.

Martin JH (1991) Autoradiographic estimation of the extent of reversible inactivation produced by microinjection of lidocaine and muscimol in the rat. Neurosci Lett 127:160-164.
Martin JH, Ghez C (1999) Pharmacological inactivation in the analysis of the central control of movement. J Neurosci Methods 86:145-159.

Nelken I, Ulanovsky N (2007) Mismatch negativity and stimulus-specific adaptation in animal models. J Psychophysiol 21:214-233.

Pérez-González D, Malmierca MS, Covey E (2005) Novelty detector neurons in the mammalian auditory midbrain. Eur J Neurosci 22:2879-2885.

Reches A, Gutfreund Y (2008) Stimulus-specific adaptations in the gaze control system of the barn owl. J Neurosci 28:1523-1533.

Rijal SO, Gross GW (2008) Dissociation constants for $\mathrm{GABA}_{\mathrm{A}}$ receptor antagonists determined with neuronal networks on microelectrode arrays. J Neurosci Methods 173:183-192.

Schwarz DW, Tennigkeit F, Puil E (2000) Metabotropic transmitter actions in auditory thalamus. Acta Otolaryngol 120:251-254.

Stiebler I, Neulist R, Fichtel I, Ehret G (1997) The auditory cortex of the house mouse: left-right differences, tonotopic organization and quantitative analysis of frequency representation. J Comp Physiol A 181:559-571.

Suga N (2008) Role of corticofugal feedback in hearing. J Comp Physiol A Neuroethol Sens Neural Behav Physiol 194:169-183.

Talwar SK, Musial PG, Gerstein GL (2001) Role of mammalian auditory cortex in the perception of elementary sound properties. J Neurophysiol $85: 2350-2358$.

Tennigkeit F, Schwarz DW, Puil E (1998) $\mathrm{GABA}_{\mathrm{B}}$ receptor activation changes membrane and filter properties of auditory thalamic neurons. Hear Res 122:18-24.

Tennigkeit F, Schwarz DW, Puil E (1999) Modulation of frequency selectivity by $\mathrm{Na}^{+}$- and $\mathrm{K}^{+}$-conductances in neurons of auditory thalamus. Hear Res 127:77-85.

Ulanovsky N, Las L, Nelken I (2003) Processing of low-probability sounds by cortical neurons. Nat Neurosci 6:391-398.

Ulanovsky N, Las L, Farkas D, Nelken I (2004) Multiple time scales of adaptation in auditory cortex neurons. J Neurosci 24:10440-10453.

von der Behrens W, Bäuerle P, Kössl M, Gaese BH (2009) Correlating stimulus-specific adaptation of cortical neurons and local field potentials in the awake rat. J Neurosci 29:13837-13849.

Winer JA (2006) Decoding the auditory corticofugal systems. Hear Res 212:1-8.

Winer JA, Larue DT (1996) Evolution of GABAergic circuitry in the mammalian medial geniculate body. Proc Natl Acad Sci U S A 93:3083-3087.

Winer JA, Larue DT, Huang CL (1999) Two systems of giant axon terminals in the cat medial geniculate body: convergence of cortical and GABAergic inputs. J Comp Neurol 413:181-197.

Yan J, Suga N (1999) Corticofugal amplification of facilitative auditory responses of subcortical combination-sensitive neurons in the mustached bat. J Neurophysiol 81:817-824.

Yan J, Zhang Y, Ehret G (2005) Corticofugal shaping of frequency tuning curves in the central nucleus of the inferior colliculus of mice. J Neurophysiol 93:71-83.

Yu XJ, Xu XX, He S, He J (2009) Change detection by thalamic reticular neurons. Nat Neurosci 12:1165-1170.

Yu YQ, Xiong Y, Chan YS, He J (2004) Corticofugal gating of auditory information in the thalamus: an in vivo intracellular recording study. J Neurosci 24:3060-3069.

Zhang Y, Suga N (1997) Corticofugal amplification of subcortical responses to single tone stimuli in the mustached bat. J Neurophysiol 78:3489-3492.

Zhang Y, Yan J (2008) Corticothalamic feedback for sound-specific plasticity of auditory thalamic neurons elicited by tones paired with basal forebrain stimulation. Cereb Cortex 18:1521-1528.

Zhang Z, Liu CH, Yu YQ, Fujimoto K, Chan YS, He J (2008) Corticofugal projection inhibits the auditory thalamus through the thalamic reticular nucleus. J Neurophysiol 99:2938-2945. 